\title{
DIRECT NUMERICAL SIMULATION FOR FLOW TRANSITION OVER \\ A FLAT PLATE
}

\author{
by \\ SHUTIAN DENG
}

\author{
Presented to the Faculty of the Graduate School of \\ The University of Texas at Arlington in Partial Fulfillment \\ of the Requirements \\ for the Degree of
}

DOCTOR OF PHILOSOPHY

THE UNIVERSITY OF TEXAS AT ARLINGTON

December 2005 
Copyright (c) by Shutian Deng 2005

All Rights Reserved 
To my lovely wife and new born baby Dawson. 


\section{ACKNOWLEDGEMENTS}

I would like to thank my supervising professor Dr. Chaoqun Liu for constantly motivating and encouraging me, and also for his invaluable advice during my doctoral studies. I wish to thank my academic advisors Dr. Guojun Liao, Dr. Jianzhong Su, Dr. Hristo V. Kojouharov, Dr. Hua shan and Dr. Qingjun Cai for their interest in my research and for taking time to serve in my dissertation committee and reviewing this paper.

I wish to thank Dr. Li Jiang, Dr. Sheng Luo, Dr. Jiangang Cai, Dr. Jiaxing Xue, Ms. Jie Liu, Mr. Peng Xie and other friends in UTA for their support and encouragement.

I would also like to extend my appreciation to HyPerComp for providing support for my doctoral studies. I am especially grateful to Dr. Zhining Liu and Dr. Ramakanth Munipali from HyPerComp for their interest, helpful discussions and invaluable comments in my research.

Thanks for High Performance Computing center at UTA providing part of the computation time.

Finally, I would like to express my deep gratitude to my parents who have encouraged and inspired me and sponsored my undergraduate and graduate studies. I am extremely fortunate to be so blessed. I am also extremely grateful to my wife for her love, care, sacrifice, encouragement and patience. I also thank several of my friends who have helped me throughout my career.

April 21, 2005 


\begin{abstract}
DIRECT NUMERICAL SIMULATION FOR FLOW TRANSITION OVER A FLAT

PLATE

Publication No.

Shutian Deng, Ph.D.

The University of Texas at Arlington, 2005
\end{abstract}

Supervising Professor: Chaoqun Liu

In this paper, direct numerical simulation (DNS) of flow transition over a flat plate at a free stream Mach number of 0.5 and a Reynolds number of 1000 based on the free stream velocity and inflow displacement thickness has been carried out. The time-dependent Navier-Stokes equations are solved directly by a third-order TVD RungeKutta method from Shu(1998). A sixth order central compact scheme from Lele (1992) that facilitates high resolution of the flow field is used for spatial discretization together with a sixth order implicit compact filter. To avoid possible non-physical wave reflection from the boundaries, the non-reflecting boundary conditions Jiang et al. (1999) are specified at the far field and the outflow boundaries. The inflow is specified by laminar flow profile with imposed eigenmodes of two-dimensional and three-dimensional TollmienSchlichting (T-S) waves and random noise. The parallel computation is accomplished through the Message Passing Interface (MPI) together with a domain decomposition approach. Computation is carried out currently in three different grids levels: $256 \times 32 \times 64$, $640 \times 64 \times 60$ and $1536 \times 128 \times 64$ in the streamwise $(\mathrm{x})$, spanwise $(\mathrm{y})$, and wall normal $(\mathrm{z})$ 
directions. In this paper, by integrating all these papers, a better view and more detail investigations about the back ground of the study, more details on different grid levels and more complete conclusions are documented. The DNS results show the mean flow properties, such as the skin friction coefficients and the mean velocity profile, wall shear linear law, log law in the turbulent region, as well as the spatial evolution of disturbance modes which agree very well with the theoretic and experimental results. Some of the structures appeared in the transition region are also studied. In addition, the statistics and spectrum analysis of the turbulence region, kinetic energy revolution and Reynolds stress are also shown in this paper. The spectra analysis shows that our resolution at the 1536x128x64 is adequate. All computational results are in good agreement with other reported work. 


\section{TABLE OF CONTENTS}

ACKNOWLEDGEMENTS .............................. iv

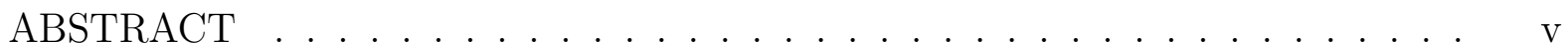

LIST OF FIGURES . . . . . . . . . . . . . . . . . . . ix

LIST OF TABLES ......................... xi

Chapter

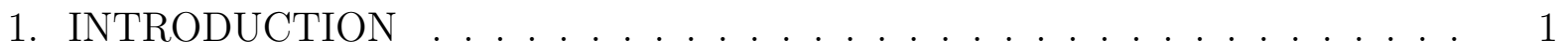

1.1 Introduction . . . . . . . . . . . . . . . . . 1

2. BACKGROUND AND LITERATURE SURVEY . . . . . . . . . . . . 4

2.1 Incompressible Laminar Flow over a Flat Plate - Blasius Solution . . . . 4

2.1.1 Boundary layer thickness . . . . . . . . . . . . . 5

2.1.2 Dimensionless analysis . . . . . . . . . . . . . . 5

2.1.3 Blasius similarity solution . . . . . . . . . . . . 6

2.2 Linear Stability Theory and Transition . . . . . . . . . . . . . . . 9

2.2.1 Linear stability of parallel viscous flow . . . . . . . . . . . 10

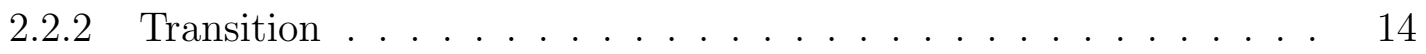

2.3 Turbulence. . . . . . . . . . . . . . . . . . . . . . . . . 18

3. GOVERNING EQUATIONS AND NUMERICAL METHODS . . . . . . . . . 23

3.1 Governing Equations . . . . . . . . . . . . . . . . . 23

3.2 Numerical Methods . . . . . . . . . . . . . . . . . . 25

3.3 Boundary Conditions . . . . . . . . . . . . . . . . 26

3.4 Parallel Computing . . . . . . . . . . . . . . . . . . 30

4. COMPUTATIONAL PROCEDURE AND RESULTS DISCUSSION . . . . . . 33 
4.1 Computational Procedure . . . . . . . . . . . . . . . 33

4.2 Computational Results . . . . . . . . . . . . . . . . . 35

4.2.1 Coarsest grids $(256 \times 32 \times 64) \ldots \ldots . \ldots . \ldots . \ldots . \ldots$

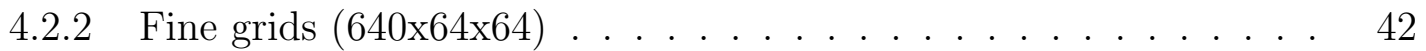

4.2.3 Finest grids $(1536 \mathrm{x} 128 \mathrm{x} 64) \ldots \ldots . \ldots . \ldots . \ldots 45$

4.3 Discussion and Comparison . . . . . . . . . . . . . . . 50

4.3.1 Skin friction comparison .............. 50

4.3.2 Velocity profiles . . . . . . . . . . . . . . 51

4.3.3 Spectra and velocity statistics .............. 53

4.3.4 Instantaneous flow field . . . . . . . . . . . . . . . . 55

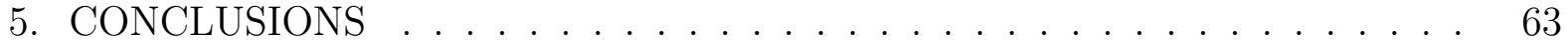
APPENDIX

A. LAMINAR FLOW OVER A FLAT PLATE . . . . . . . . . . . . . . . . 65

REFERENCES . . . . . . . . . . . . . . . . . . . . 74

BIOGRAPHICAL STATEMENT . . . . . . . . . . . . . . . . 78 


\section{LIST OF FIGURES}

Figure $\quad$ Page

2.1 Velocity profile from Blasius similarity solution . . . . . . . . . . . 8

2.2 Relative stability . . . . . . . . . . . . . . . . . . . 9

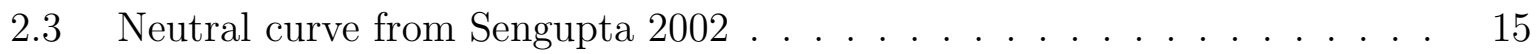

3.1 The domain decomposition along $\xi$ direction . . . . . . . . . . . . . 31

3.2 The change of data structure for calculating derivative in $\xi$ direction . . . 32

4.1 Computation domain . . . . . . . . . . . . . . . . 33

4.2 Case 1 instantaneous flow field . . . . . . . . . . . . . . 36

4.3 Case 2 spatial growth of streamwise perturbation . . . . . . . . . . 38

4.4 Case 2 skin friction comparison . . . . . . . . . . . . 38

4.5 Case 2 instantaneous flow field . . . . . . . . . . . . . . . . 39

4.6 Case 3 spatial growth of streamwise perturbation . . . . . . . . . . . 41

4.7 Case 3 skin friction comparison . . . . . . . . . . . . . . 41

4.8 Case 3 instantaneous flow field . . . . . . . . . . . . . . . . 42

4.9 Case 4 spatial growth of streamwise perturbation . . . . . . . . . . 43

4.10 Case 4 skin friction comparison . . . . . . . . . . . . . 44

4.11 Case 4 instantaneous flow field . . . . . . . . . . . . . . . . 44

4.12 Case 5 spatial growth of streamwise perturbation . . . . . . . . . . 46

4.13 Case 5 skin friction comparison . . . . . . . . . . . . . 46

4.14 Case 5 instantaneous flow field . . . . . . . . . . . . . . . 47

4.15 Case 6 spatial growth of streamwise perturbation . . . . . . . . . . . 48

4.16 Case 6 skin friction comparison . . . . . . . . . . . . . . . . . 49 
4.17 Case 6 instantaneous flow field . . . . . . . . . . . . . . . . 49

4.18 Velocity profiles for Case $2 \ldots \ldots \ldots$. . . . . . . . . . . 51

4.19 Velocity profiles for Case $4 \ldots \ldots$. . . . . . . . . . . . 52

4.20 Velocity profiles for Case 5 . . . . . . . . . . . . . . . . . 52

4.21 Velocity profiles for Case $6 \ldots \ldots$. . . . . . . . . . . . 53

4.22 Log-linear plots for Case $2 \ldots \ldots$. . . . . . . . . . . . 54

4.23 Log-linear plots for Case $4 \ldots \ldots$. . . . . . . . . . . . . 55

4.24 Log-linear plots for Case $5 \ldots \ldots$. . . . . . . . . . . . . 56

4.25 Log-linear plots for Case $6 \ldots \ldots$. . . . . . . . . . . 57

4.26 Spectra in $\mathrm{x}$ direction $($ Case 5) . . . . . . . . . . . . 58

4.27 Spectra in $\mathrm{x}$ direction $($ Case 5) . . . . . . . . . . . . . 58

4.28 Spectra in $\mathrm{x}$ direction $($ Case 6 ) . . . . . . . . . . . . . 59

4.29 Spectra in $\mathrm{x}$ direction $($ Case 6) . . . . . . . . . . . . . . 59

4.30 Reynolds stress (Case 5) . . . . . . . . . . . . . . . 60

4.31 Reynolds stress $($ Case 6$)$. . . . . . . . . . . . . . . . 60

4.32 Instantaneous flow field $($ Case 2$) \ldots \ldots$. . . . . . . . . . . 61

4.33 Instantaneous flow field $($ Case 4$) \ldots \ldots$. . . . . . . . . . 61

4.34 Instantaneous flow field $($ Case 5$) \ldots \ldots$. . . . . . . . . . 62

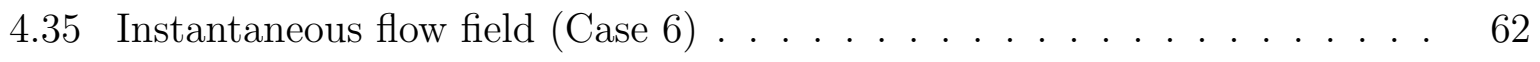




\section{LIST OF TABLES}

Table $\quad$ Page

4.1 Flow parameters ....................... 34

4.2 Typical inflow disturbance parameters . . . . . . . . . . . . . . 35

4.3 Case 1 inflow parameters of coarsest grids . . . . . . . . . . . . . 36

4.4 Case 2 inflow parameters of coarsest grids . . . . . . . . . . . . . . 37

4.5 Case 3 inflow parameters of coarsest grids . . . . . . . . . . . . . . 40

4.6 Case 4 inflow parameters of finer grids . . . . . . . . . . . . . . . 43

4.7 Case 5 inflow parameters of finest grids . . . . . . . . . . . . . 45

4.8 Case 6 inflow parameters of finest grids . . . . . . . . . . . . . . . . 48

5.1 Inflow disturbance parameters and transition type . . . . . . . . . 63 


\section{CHAPTER 1 \\ INTRODUCTION}

\section{$1.1 \quad$ Introduction}

The main driving force for the study of flow transition is the understanding, prediction and control of transition to turbulence. The transition process from laminar to turbulent flow in a shear layer is a basic scientific problem in modern fluid mechanics and has been the subject of study for over a century.

For the past century, numerous investigations have been conducted in an attempt to predict the transition from laminar to turbulent flow in boundary layers. Orr and Sommerfeld accomplished the early theoretical contributions independently at the beginning of the 20th century. Their achievement was based on linearized disturbance equations, which were referred to the Orr-Sommerfeld equations. About 20 years later, Tollmien was first able to solve the Orr-Sommerfeld equation and Schlichting computed amplification rates of disturbances in the boundary layer. The experimental confirmation of the classical hydrodynamic stability theory was first given by Schubauer and Skramstad.

Nonlinear stability, starting with the formation of three-dimensional structures, is also referred as the secondary instability. The aligned three-dimensional structure associated with the peak valley splitting of secondary instability was first measured in detail by Klebanoff, Tidstrom and Sargent (1962). This type of secondary instability is now referred as fundamental or K-type after Klebanoff. Later in boundary layer experiments, another type of secondary instability characterized by subharmonic signals and reveals staggered patterns of three-dimensional structure is found by Kachanov(1978). These experiments showed that the subharmonic of the fundamental wave was excited by the 
boundary layer. At small amplitude, it produced the resonant wave interaction as predicted by Craik (1971). This type of instability is referred as C-type after Craik. At larger amplitude, Craik's mechanism becomes less important and the instability is characterized by the dominance of off-resonance mode. This type of instability is referred to as H-type after Herbert (1988).

Direct numerical simulation (DNS) / large eddy simulation (LES) of flow transition over a flat plate can provide a detailed insight into the physical phenomena and some helps to interpret experiments, and has become a very important partner besides experiment and modern analysis for the study of flow transition. But due to the limitation of computation resources, the majority of the early investigations were using the temporal approach. Among them are Orszag and Patera (1983), Laurien and Kleiser (1989), temporal DNS Erlebacher and Hussaini (1990), temporal LES El-hady and Zang (1995). Temporal simulations follow the time evolution of a single wavelength of the disturbance as it convects with the phase speed of the wave. Available mesh resolution can then be focused on resolving a single wavelength. This enables simulation into the later stage of transition and reveals good agreement with the experiments. However, the assumption of periodicity along the streamwise direction does not allow direct comparison of temporal numerical simulations with spatially evolving experimental results. Moreover, streamwise growth of the base flow (in boundary layers) is not allowed in the temporal approach. Hence, boundary layer simulations are restricted to the parallel flow assumption as well. This approach basically lacks a physically realistic representation.

The recent development in computer technology and numerical methods has enabled the application of the spatial DNS/LES approaches, which can provide the needed quantitative information that comparable to the realistic flow transition (Ducros et al. 1996; Liu et al. 1995). But with spatial DNS/LES, there still exists some difficulties, among which are the realistic specifications of inflow and outflow boundary conditions 
and the high demands on the computational resources. To simulate transition from laminar to turbulent flow, the inflow boundary condition can be specified as the composition of the laminar flow profile and two- and three-dimensional perturbations. For subsonic flow, the non-reflecting boundary conditions (Jiang et al. 1999) can be used for far field and outflow boundaries. The high demands on computational resources are solved by using parallel computation, as shown by some previous works based on domain decomposition and MPI (Jiang et al. 2001).

In the present work, the spatial transition of the flat plate flow at a Mach number of 0.5 is studied by parallel DNS based on the high-order compact scheme and a third order TVD Runge-Kutta method. The paper is organized as follows: Chapter 1 presents the brief introduction. Chapter 2 gives a detailed overview of the subject and literature survey. Chapter 3 has the governing equation and numerical method used. Simulation results and discussions can be found in Chapter 4. Finally, Chapter 5 gives concluding remarks. 


\section{CHAPTER 2}

\section{BACKGROUND AND LITERATURE SURVEY}

\subsection{Incompressible Laminar Flow over a Flat Plate - Blasius Solution}

Laminar flow is a type of fluid flow in which the fluid travels smoothly or in regular paths. It is quite different from turbulent flow, in which the fluid undergoes random fluctuations and strong mixing. Laminar flow actually is not common at all. It only happens in cases in which the flow channel is relatively small, the fluid is moving slowly, and its viscosity is relatively high. Some examples include oil flow through a thin tube, blood flow through capillaries and thick mud flowing downhill. Most other kinds of fluid flows are turbulence except for one near solid boundaries, where the flow is often laminar, especially in a thin layer just adjacent to the solid surface. In laminar flow, sometimes called streamline flow, the velocity, pressure and other flow properties at each point in the fluid remain constant. Laminar flow over a horizontal surface may be thought of as consisting of thin layers all parallel to each other. The fluid in contact with the horizontal surface has the same a velocity with the wall for a viscous flow, and all the rest layers slide over each other. For viscous flow, at large Reynolds numbers, the effect of viscosity is limited to a very thin layer near the wall. That will leave the mainstream far away from the wall irrotational. This means that the rate of the convection is much larger than the rate of viscous diffusion. Thus a thin boundary layer will present. In the case of a flat plate, for Reynolds number in the range of $\left[1000,10^{5}\right]$, the boundary layer will be laminar; for Reynolds number $10^{6}$, it is likely to be turbulent. In the rest of this section, some very basic properties of laminar flow will be introduced. 


\subsubsection{Boundary layer thickness}

Let's consider viscous flow over a flat plate. At the wall, the velocity is zero. As the distance to the wall increases, the velocity increases as well. When the distance reaches a certain height, say $\delta$, the velocity equal to the freestream velocity. This height is call boundary layer thickness. From practical point of view, when the velocity reaches $99 \%$ of freestream velocity, the height is defined as the boundary layer thickness.

There are other classical boundary layer thickness definitions:

Displacement thickness: $\delta^{*}=\int_{0}^{y \rightarrow \infty}\left(1-\frac{u}{U}\right) d y$

Momentum thickness: $\theta=\int_{0}^{y \rightarrow \infty} \frac{u}{U}\left(1-\frac{u}{U}\right) d y$

One of the well known estimations is:

$$
\frac{\delta}{x} \approx \frac{5.0}{\sqrt{R e_{x}}}, \frac{\delta^{*}}{x} \approx \frac{1.72}{\sqrt{R e_{x}}}, \frac{\theta}{x} \approx \frac{0.664}{\sqrt{R e_{x}}}
$$

More details can be found in appendix A.

\subsubsection{Dimensionless analysis}

Suppose we try to find a simple expression for velocity profile $\mathrm{u}(\mathrm{y})$ at any position x. Physically, there are three conditions we need:

$$
\begin{aligned}
u(0) & =0 \\
u(\delta) & =U \\
\left.\frac{\partial u}{\partial y}\right|_{y=0} & =0
\end{aligned}
$$

A good approximation could be a second order polynomial as follows

$$
u=U\left(\frac{2 y}{\delta}-\frac{y^{2}}{\delta^{2}}\right)
$$


For a two dimensional incompressible flow, the full Navier-Stokes equations can be written as follows:

$$
\begin{array}{r}
\frac{\partial u}{\partial x}+\frac{\partial v}{\partial y}=0 \\
\frac{\partial u}{\partial t}+u \frac{\partial u}{\partial x}+v \frac{\partial u}{\partial y}=-\frac{1}{\rho} \frac{\partial p}{\partial x}+\nu\left(\frac{\partial^{2} u}{\partial x^{2}}+\frac{\partial^{2} u}{\partial y^{2}}\right) \\
\frac{\partial v}{\partial t}+u \frac{\partial v}{\partial x}+v \frac{\partial v}{\partial y}=-\frac{1}{\rho} \frac{\partial p}{\partial y}+\nu\left(\frac{\partial^{2} v}{\partial x^{2}}+\frac{\partial^{2} v}{\partial y^{2}}\right)
\end{array}
$$

The importance of boundary layer is that the layer is very thin, that is $\delta \ll x$. It is true when Re is large, where $\mathrm{x}$ has the same scale as the flat plate length. For laminar boundary layer we can also see that $v \ll u$. Also by differentiating Eq. 2.1, we know that $\frac{\partial u}{\partial x} \ll \frac{\partial u}{\partial y}$ and $\frac{\partial v}{\partial x} \ll \frac{\partial v}{\partial y}$. From these reasonable estimates, Prandtl derived the famous boundary layer equations. By redefining all variables in terms of these estimates, for a flow with large Reynolds number, we can eliminate the terms that are negligible from the full equation. Following are the simplified equations for a low speed steady flow boundary layer when we only consider the continuity and momentum equation:

$$
\begin{array}{r}
\frac{\partial u}{\partial x}+\frac{\partial v}{\partial y}=0 \\
u \frac{\partial u}{\partial x}+v \frac{\partial u}{\partial y}=\nu \frac{\partial^{2} u}{\partial y^{2}}
\end{array}
$$

This is a system of parabolic type partial differential equations. It is much simpler than the original Navier-Stokes equations.

\subsubsection{Blasius similarity solution}

Starting from Eq. 2.5, one can try to solve the partial differential equations using methods such as finite difference. H. Blasius is the one came up with the idea of reducing the partial differential equations even further into an ordinary differential equations by 
similarity transformation. Let us transform the independent variables $(\mathrm{x}, \mathrm{y})$ to $(\xi, \eta)$, where

$$
\xi=x \text { and } \eta=y \sqrt{\frac{V_{\infty}}{\nu x}}
$$

Using the chain rule, we have the following:

$$
\begin{array}{r}
\frac{\partial}{\partial x}=\frac{\partial}{\partial \xi} \frac{\partial \xi}{\partial x}+\frac{\partial}{\partial \eta} \frac{\partial \eta}{\partial x} \\
\frac{\partial}{\partial y}=\frac{\partial}{\partial \xi} \frac{\partial \xi}{\partial y}+\frac{\partial}{\partial \eta} \frac{\partial \eta}{\partial y} \\
\frac{\partial^{2}}{\partial y^{2}}=\frac{V_{\infty}}{\nu x} \frac{\partial^{2}}{\partial \eta^{2}}
\end{array}
$$

Also consider the stream function of the flow, $\psi=\left.\int u d y\right|_{x=c o n s t}$ should increase as $\delta$ increases, it has the following nondimensional form:

$$
\psi=\sqrt{\nu V_{\infty} x} f(\eta)
$$

where $\mathrm{f}$ is the unknowns function of $\eta$ only. This definition of $\psi$ satisfies continuity equation, so the stream function definition is legitimate.

$$
\begin{gathered}
u=\frac{\partial \psi}{\partial y}=\sqrt{\frac{V_{\infty}}{\nu x}} \frac{\partial \psi}{\partial \eta}=V_{\infty} f^{\prime}(\eta) \\
v=-\frac{\partial \psi}{\partial x}=\left(\frac{\partial \psi}{\partial \xi}+\frac{\partial \psi}{\partial \eta} \frac{\partial \eta}{\partial x}\right)=-\frac{1}{2} \sqrt{\frac{\nu V_{\infty}}{x}} f-\sqrt{\nu x V_{\infty}} \frac{\partial \eta}{\partial x} f^{\prime}
\end{gathered}
$$

Substitute Eq. 2.7 to Eq. 2.12 into momentum equation Eq. 2.5, expanded out

$$
V_{\infty} f^{\prime}\left(V_{\infty} \frac{\partial \eta}{\partial x} f^{\prime \prime}\right)-\left(\frac{1}{2} \sqrt{\frac{\nu V_{\infty}}{x}} f+\sqrt{\nu x V_{\infty}} \frac{\partial \eta}{\partial x} f^{\prime}\right) V_{\infty} \sqrt{\frac{V_{\infty}}{\nu x}} f^{\prime \prime}=\nu V_{\infty} \frac{V_{\infty}}{\nu x} f^{\prime \prime}
$$

Simplifying that, we have

$$
V_{\infty}^{2} \frac{\partial \eta}{\partial x} f^{\prime} f^{\prime \prime}-\frac{1}{2} \frac{V_{\infty}^{2}}{x} f f^{\prime \prime}-V_{\infty}^{2} \frac{\partial \eta}{\partial x} f^{\prime} f^{\prime \prime}=\frac{V_{\infty}^{2}}{x} f^{\prime \prime \prime}
$$

Finally, after get rid of all the extra terms

$$
2 f^{\prime \prime \prime}+f f^{\prime \prime}=0
$$


This is the famous Blasius's equation. The flat plate solution using Eq. 2.15 is the first application of the boundary layer theory. This is an ordinary differential equation for $f(\eta)$, and it also defines velocity by Eq. 2.12. For a nonlinear, third order ordinary differential equation like this, many standard numerical method can be used to solve it, namely Runge-Kutta method. A shooting procedure is also required for solving numerically. Since we need three boundary conditions specified at the wall, but there are only two boundary conditions are known from the variables transformation:

At $\eta=0: \quad f=0, f^{\prime}=0$

So a third condition $f^{\prime \prime}$ must be assumed at $\eta=0$, and then we do the integration to check if $\mathrm{f}^{\prime}$ at a large $\eta$ match $f^{\prime}=1$ or not. If yes, the calculation is finished; if not, we need to make another guess and "shoot" again. This is a classical approach for solving ordinary differential equations. The solution of Eq. 2.15 is plotted in Fig. 2.1.



Figure 2.1 Velocity profile from Blasius similarity solution 


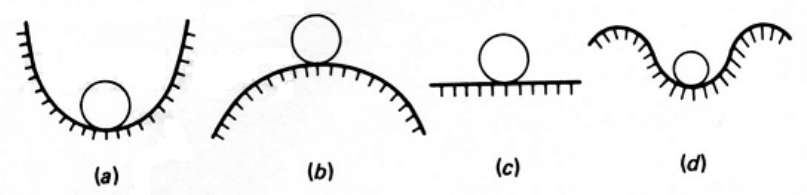

Figure 2.2 Relative stability

\subsection{Linear Stability Theory and Transition}

In this section, we will talk about stability and transition. The general stability concept is to test how one system reacts to disturbance. From Fig. 2.2, we can see that there are four scenarios:

- Stable

- Unstable

- Neutral stability

- Stable for small disturbance but unstable for large disturbance

In the case of boundary-layer flow, the stability analysis is somewhat complicated. For a small disturbance, the flow maybe stable, but a large enough disturbance can cause the flow to become turbulent. Stability analysis is to show the effect of a particular disturbance. In viscous flow, it is well known that above a large enough Reynolds number, laminar flow becomes unstable. Transition describes the change from laminar to turbulence over time and space. There are very limited theoretical results available for transition so far. The linearized stability theory is available to predict the spatial amplification rates. Lots of empirical predictions from experiments are also available. 


\subsubsection{Linear stability of parallel viscous flow}

The stability analysis has fundamental importance in both practical and theoretical point of view. In most cases, the transition to turbulence starts as a result of instability to small perturbations. Typically, viscous flow instability study is carried out as follows

- Select a basic solution flow and add a disturbance

- Find the disturbance equations and linearize

- Simplify the problem to a eigenvalue problem

- Solve the problem and draw neutral curves

In this case, it is possible to simplify the problem of stability. We can analyze the linearized equations of disturbances by reducing the complexity of non-linear motion equation. The classical approach to the solution of such stability problems is the method of normal modes, consisting of a reduction of the linear initial-boundary-value problem to a eigenvalues problem. Consider the Navier-Stokes equation:

$$
\begin{array}{r}
\frac{\partial u}{\partial x}+\frac{\partial v}{\partial y}+\frac{\partial w}{\partial z}=0 \\
\frac{\partial u}{\partial t}+u \frac{\partial u}{\partial x}+v \frac{\partial u}{\partial y}+w \frac{\partial u}{\partial z}=-\frac{1}{\rho} \frac{\partial p}{\partial x}+\nu\left(\frac{\partial^{2} u}{\partial x^{2}}+\frac{\partial^{2} u}{\partial y^{2}}+\frac{\partial^{2} u}{\partial z^{2}}\right) \\
\frac{\partial v}{\partial t}+u \frac{\partial v}{\partial x}+v \frac{\partial v}{\partial y}+w \frac{\partial v}{\partial z}=-\frac{1}{\rho} \frac{\partial p}{\partial y}+\nu\left(\frac{\partial^{2} v}{\partial x^{2}}+\frac{\partial^{2} v}{\partial y^{2}}+\frac{\partial^{2} v}{\partial z^{2}}\right) \\
\frac{\partial w}{\partial t}+u \frac{\partial w}{\partial x}+v \frac{\partial w}{\partial y}+w \frac{\partial w}{\partial z}=-\frac{1}{\rho} \frac{\partial p}{\partial z}+\nu\left(\frac{\partial^{2} w}{\partial x^{2}}+\frac{\partial^{2} w}{\partial y^{2}}+\frac{\partial^{2} w}{\partial z^{2}}\right)
\end{array}
$$

For flat plate, we can get the basic flow solution by solving Blasius Equation (Eq. 2.15). Denote the solution as $(U, V, W, P)$. To find out whether these are stable solutions, we add on small perturbation $(\hat{u}, \hat{v}, \hat{w}, \hat{p})$ and substitute the superimposed variables $(U+$ $\hat{u}, V+\hat{v}, W+\hat{w}, P+\hat{p})$ into Eq. (2.19), then we eliminate the original equalities containing 
$(U, V, W, P)$ and neglect higher powers and products of $(\hat{u}, \hat{v}, \hat{w}, \hat{p})$. Thus we will end up with

$$
\begin{aligned}
\frac{\partial \hat{u}}{\partial x}+\frac{\partial \hat{v}}{\partial y}+\frac{\partial \hat{w}}{\partial z} & =0 \\
\frac{\partial \hat{u}}{\partial t}+U \frac{\partial \hat{u}}{\partial x}+\hat{u} \frac{\partial U}{\partial x}+V \frac{\partial \hat{u}}{\partial y}+\hat{v} \frac{\partial U}{\partial y}+W \frac{\partial \hat{u}}{\partial z}+\hat{w} \frac{\partial U}{\partial z} & = \\
-\frac{1}{\rho} \frac{\partial \hat{p}}{\partial x}+\nu\left(\frac{\partial^{2} \hat{u}}{\partial x^{2}}+\frac{\partial^{2} \hat{u}}{\partial y^{2}}+\frac{\partial^{2} \hat{u}}{\partial z^{2}}\right) & = \\
\frac{\partial \hat{v}}{\partial t}+U \frac{\partial \hat{v}}{\partial x}+\hat{u} \frac{\partial V}{\partial x}+V \frac{\partial \hat{v}}{\partial y}+\hat{v} \frac{\partial V}{\partial y}+W \frac{\partial \hat{v}}{\partial z}+\hat{w} \frac{\partial V}{\partial z} & = \\
\left.\frac{\partial \hat{w}}{\partial t}+U \frac{\partial \hat{w}}{\partial x}+\hat{\rho} \frac{\partial W}{\partial x}+V \frac{\partial \hat{w}}{\partial y}+\hat{v} \frac{\partial W}{\partial y}+W \frac{\partial^{2} \hat{v}}{\partial x^{2}}+\frac{\partial^{2} \hat{v}}{\partial y^{2}}+\frac{\partial^{2} \hat{v}}{\partial z^{2}}\right) & =\hat{\frac{\partial W}{\partial z}}= \\
-\frac{1}{\rho} \frac{\partial \hat{p}}{\partial z}+\nu\left(\frac{\partial^{2} \hat{w}}{\partial x^{2}}+\frac{\partial^{2} \hat{w}}{\partial y^{2}}+\frac{\partial^{2} \hat{w}}{\partial z^{2}}\right) &
\end{aligned}
$$

The above equations are linear partial differential equations for $(\hat{u}, \hat{v}, \hat{w}, \hat{p})$, and $(U, V, W, P)$ are the given basic flow. These equations can be further simplified to a single ordinary differential equation by assuming a locally parallel basic flow. If y is the wall normal direction, we can think of that the velocity $\mathrm{V}$ is very small. Moreover, we assume that $\mathrm{U}, \mathrm{W}$ are only functions of $\mathrm{y}$. This will eliminate more convective terms on the left hand sides.

As the most general form of three dimensional disturbance is that of a travelling wave whose amplitude varies with $\mathrm{y}$ and move along the wall at an angle $\gamma$. Where $\gamma=\arctan \left(\frac{\beta}{\alpha}\right)$ is the angle between the direction of propagation of wave and the streamwise direction. Suppose that the full solution can be expressed as a sum of elementary solution(modes), which has the form

$$
\hat{u}(y), \hat{v}(y), \hat{w}(y), \hat{p}(y)=[u(y), v(y), w(y), p(y)] e^{i[(\kappa \gamma)-\omega t]}
$$

where $\hat{u}(y), \hat{v}(y), \hat{w}(y), \hat{p}(y)$ are complex amplitude functions of the disturbances; $\kappa$ is a wave vector with $\kappa=\alpha^{2}+\beta^{2}$, where $\alpha$ and $\beta$ are (generally complex) wave numbers in 
streamwise and spanwise directions (normally that means $\mathrm{x}$ and $\mathrm{z}$ directions); $\omega$ is the circular frequency of the disturbance and $i=\sqrt{-1}$. Another way is to let

$$
\hat{u}(y), \hat{v}(y), \hat{w}(y), \hat{p}(y)=[u(y), v(y), w(y), p(y)] e^{i \alpha[(x \cos \gamma+z \sin \gamma)-c t]}
$$

That is the disturbance which has wave number $\alpha$, propagation speed c and frequency $\omega=\alpha c$. These waves are call Tollmien-Schlichting waves historically. If we put Eq. (2.21) into Eq. (2.20), following are the results:

$$
\begin{array}{r}
i \alpha u \cos \gamma+v^{\prime}+i \alpha w \sin \gamma=0 \\
i \alpha u(U \cos \gamma+W \sin \gamma-c)+U^{\prime} v=-\frac{i}{\rho} \alpha p \cos \gamma+\nu\left(u^{\prime \prime}-\alpha^{2} u\right) \\
i \alpha v(U \cos \gamma+W \sin \gamma-c)=-\frac{i}{\rho} p^{\prime}+\nu\left(v^{\prime \prime}-\alpha^{2} v\right) \\
i \alpha w(U \cos \gamma+W \sin \gamma-c)+W^{\prime} v=-\frac{i}{\rho} \alpha p \sin \gamma+\nu\left(w^{\prime \prime}-\alpha^{2} w\right)
\end{array}
$$

We may assume that the perturbation grow either spatially ( $\alpha$ complex and $\alpha c$ real) or else temporally ( $\alpha$ real and $\alpha c$ complex). Define

$$
\begin{array}{r}
u_{0}=u \cos \gamma+w \sin \gamma \\
U_{0}=U \cos \gamma+W \sin \gamma
\end{array}
$$

By multiplying Eq. (2.23) with $\cos \gamma$ and Eq. (2.25) with $\sin \gamma$ and sum up, we end up with

$$
\begin{aligned}
i \alpha u_{0}+v^{\prime} & =0 \\
i \alpha u_{0}\left(U_{0}^{\prime}-c\right)+U^{\prime} v & =-\frac{i}{\rho} \alpha p^{\prime}+\nu\left(u_{0}^{\prime \prime}-\alpha^{2} u_{0}\right) \\
i \alpha v\left(U_{0}^{\prime}-c\right) & =-\frac{i}{\rho} p^{\prime}+\nu\left(v^{\prime \prime}-\alpha^{2} v\right)
\end{aligned}
$$

These are two dimensional equations whose components $u_{0}$ and $U_{0}$ are parallel to the direction of propagation $\gamma$ of the travelling waves. Thus the stability of any parallel 
flow along any direction $\gamma$ can be found from a two dimensional analysis for the effective basic flow $U_{0}(y)$ along that direction. Further if $\mathrm{W}$ is zero as if the basic flow is purely two dimensional. Then the shape of $U(y)$ is independent of $\gamma$. Therefore, for an oblique disturbance (in this paper refer to three dimensional disturbance), the basic flow $U \cos (\gamma)$ is smaller and is more stable than when the disturbance propagate parallel to $U(y)$. This result is contained in the Squire Theorem.

Squire's Theorem For a two dimensional parallel flow $U(y)$, the minimum critical unstable Reynolds's number occurs for the case of a two dimensional disturbance propagate along the same direction $(\gamma=0)$.

So we know that for three dimensional waves are having smaller effective Reynolds number than the corresponding two dimensional waves (with $\gamma=0$ ). That is saying that two dimensional modes are more unstable for the same base flow (this is true for low Reynolds number). From this point on it is convenient to consider only the two dimensional waves. If we manipulate Eq. (2.26) and eliminate $u$ and $v$, we get an Poisson equation regarding pressure

$$
p^{\prime \prime}-\alpha^{2} p=-2 i \alpha U^{\prime} v
$$

Its source term is proportional to the product $\frac{\partial U}{\partial u} \frac{\partial \hat{v}}{\partial x}$. In the freestream, where $U^{\prime}=0$, the solution should be in the form of $p=a e^{-\alpha y}+b e^{\alpha y}$. As disturbance vanishes at freestream, $b=0$. So $p=a e^{-\alpha y}$ and it damps exponentially.

It is relatively more involved to eliminate $v$ and $p$ from Eq. 2.26. If we want to eliminate $u$ and $p$, this will result in the Orr-Sommerfeld equation as following

$$
(U-c)\left(v^{\prime \prime}-\alpha^{2} v\right)-U^{\prime \prime} v+\frac{i v}{\alpha}\left(v^{\prime \prime \prime \prime}-2 \alpha^{2} v^{\prime \prime}+\alpha^{4} v\right)=0
$$

This equation is first derived independently by Orr (1907) and Sommerfeld (1908). Boundary conditions for this equation for boundary layer flow are $v(0)=v^{\prime}(0)=$ $0, v(\infty)=v^{\prime}(\infty)=0$. 
From the above we know that the Orr-Sommerfeld equation and its boundary conditions are homogeneous. Thus we are looking at a eigenvalues problem for the normal velocity of the disturbance $v$. It has three parameters: $\alpha, c$ and $\nu$ (or use Reynolds number $\left.R e=\frac{U \delta}{\nu}\right)$. For a given profile $U(y)$ and $U^{\prime \prime}(y)$, only a certain continuous but limited sequence of these parameters(the eigenvalues) will satisfy the equation and boundary condition. The purpose of stability analysis is to find this sequence, which has a different functional form for spatial and temporal disturbance growth

$$
\begin{aligned}
\text { Spatial growth } f\left(\operatorname{Re}, \alpha_{r}, \alpha_{i}, \omega\right) & =0 \\
\text { Temporal growth } f\left(\operatorname{Re}, \alpha, c_{r}, c_{i}\right) & =0
\end{aligned}
$$

The case of neutral stability is of interest: for temporal case $c_{i}=0$ and for spatial case $\alpha_{i}=0$. The job of stability analysis is to find the neutral points. Based on the two dimensional Orr-Sommerfeld equation solution, for a flat plate boundary layer, typical results of linear stability calculation of the neutral points are shown as follows in Fig. 2.3 .

Tollmien(1929) is the first one predicted instability for an incompressible flow. Schlichting(1933) then carried out a detailed calculation of the characteristic of the oscillations of the instability. By experiment, Schubauer and Skramstad(1947) validate the prediction given by Tollmien and Schlichting, which shows the experimental data agree well with the amplitude distribution of the oscillations calculated by Schlichting. In 1944, Lin reproduced the neutral curve proposed by Schlichting.

\subsubsection{Transition}

Laminar-to-turbulent transition is a non-linear process, there is no complete understanding or well established theory available. Parameters such as skin friction, kinetic energy, heat transfer rates and velocity profile are different dramatically between laminar 


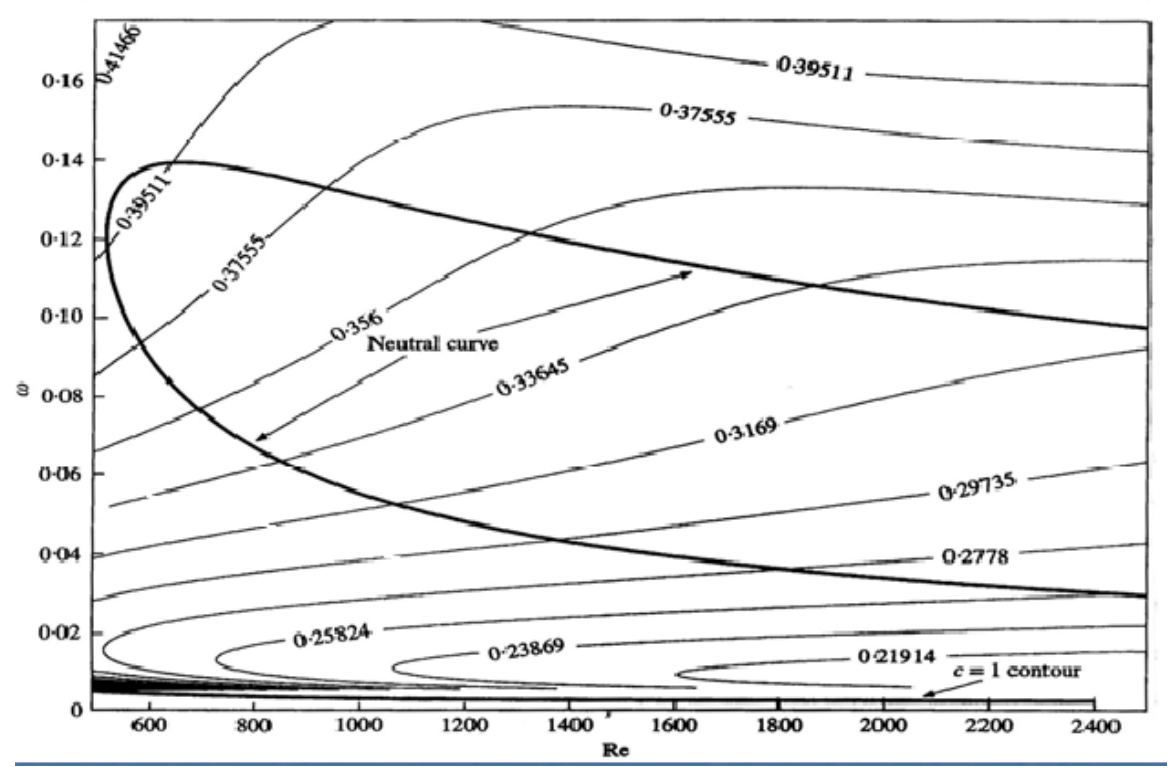

Figure 2.3 Neutral curve from Sengupta 2002

and turbulent boundary layers. Practical applications include controllability of airfoils and high-lift devices, turbine and engine performance, and aircraft and spacecraft performance. For some cases, the drag coefficient for a turbulence flow can be $60 \%$ higher than the laminar flow. People want to find a way to delay transition in these cases. Another consideration is to make transition happen earlier so as to avoid the adversed flow and stalling. A fundamental understanding of the laminar-to-turbulent transition process can lead to improved transition prediction techniques and eventually transition control technique.

Transition is usually initiated by the amplification of unstable instability waves mentioned above as Tollmien-Schlichting (T-S) waves - through receptivity to the environmental disturbances. The stability of parallel laminar flow with two-dimensional small disturbances is described by the primary linear stability theory. When the primary unstable wave is amplified to a critical threshold, it becomes unstable to three-dimensional disturbances as a result of secondary instability. At later stages of the transition, these 
new mechanisms produce three-dimensional vortex structures, which lead to breakdowns. It is well acknowledged that the type of transition depends on the amplitude of the initial disturbances and the background noise characteristics. Generally, we categorize transitions into these two types(K-type and H-type). There are other transition type as mixed and bypass. In the transition flow, linear growth of the primary wave evolves rapidly to transition through nonlinear interactions. Once they are initiated, nonlinear processes in the transition become violent and turbulent. As a consequence, it becomes very difficult to control the flow field effectively. Hence, it is of importance to understand the physics of linear instability for scenario needs to control the flow instability in the near non-linear range, see Shan (2005) .

It is well known that the three-dimensional disturbances (naturally from background noise leads to secondary instability) is necessary for the laminar-to-turbulent transition process (Klebanoff and Tidstrom 1959; Klebanoff, Tidstrom and Sargent 1962). From our numerical experiment, same conclusions are obtained. If there is only a twodimensional disturbance imposed at the inflow, then all we observe is the linear growth of the T-S waves. After certain distance, when the Reynolds number increases, the two-dimensional disturbance dies down (see the neutral curve). The breakdown scenario observed by Klebano et al. is referred to as fundamental or K-type breakdown and begins with the amplification of initially two-dimensional Tollmien-Schlichting (TS) waves. As these primary (T-S) waves exceed a threshold value of typically $1 \%$ of the free-stream velocity, three-dimensional structures evolve with spanwise periodicity of alternating peaks and valleys and result in an aligned peak-valley structure as K-type transition. Using Floquet theory of secondary instability, Herbert (1985) showed that for large initial two-dimensional T-S amplitudes relative to random background disturbances, primary resonance occurs leading to K-type breakdown. Subharmonic breakdown is another type of transition. It is characterized by a staggered peakvalley struc- 
ture and was first observed by Knapp and Roache (1968). Three-dimensional excitation derived from the background disturbance with controlled subharmonic spectral energy have been experimentally observed on flat-plate boundary layers (e.g. Kachanov, Kozlov and Levchenko 1977; Saric, Kozlov and Levchenko 1984; Saric and Thomas 1984; Kachanov and Levchenko 1984; Corke and Mangano 1989). Subharmonic breakdown is characterized by small two-dimensional T-S amplitudes (typically less than $0.8 \%$ of the free-stream velocity) and produce either a C-type (see Craik 1971) or H-type (see Herbert 1988) breakdown, where H-type is the more general type of subharmonic instability that occurs for a broad band of spanwise wavenumbers as a result of parametric resonance. The evolution of wavepackets in laminar boundary layers has also been studied as a model of natural transition to turbulence since a wavepacket produces a broad spectrum of frequencies and spanwise wavenumbers. The evolution of a localized disturbance in a laminar boundary layer from a low amplitude wavepacket to the formation of a turbulent spot has been experimentally studied by Cohen, Breuer and Haritonidis (1991) and Breuer, Cohen and Haritonidis (1997). In the linear stage of the wavepacket evolution, the wavenumber-frequency spectra indicated that most of the energy was concentrated in two-dimensional modes centered about a fundamental frequency corresponding to the most-amplified mode according to linear stability theory (LST). As nonlinear effects evolve in the wavepacket, increased energy corresponding to oblique modes at a frequency one half of the fundamental (subharmonic modes) were observed to be dominant in the wavenumber-frequency spectra. The spanwise wavenumber corresponding to these oblique modes suggested that the modes grew as a subharmonic resonance triad as described by Craik. The transition scenarios discussed above all start with strong twodimensional modes, but as the three-dimensional modes gain energy, transition advances rapidly. The selection process of boundary layers has identified oblique modes as an essential ingredient for the onset of laminar-to-turbulent breakdown. Recognizing that direct 
seeding of oblique modes may be very efficient as a transition promoter. Schmid and Henningson (1992), using direct numerical simulations (temporal) applied to a channel flow, investigated a transition mechanism involving a pair of oblique O-S modes $(\omega, \pm \beta)$ in the frequency spanwise-wavenumber space, the so-called oblique transition. They found that this transition scenario occurred at a much faster time scale than the one for secondary instability, assuming the same input disturbance energy for both scenarios. They also found that nonlinear interactions were mainly responsible for the initial energy transfer to other spanwise modes that then grew by linear mechanisms. The dominance of the linear transfer mechanism was reduced as transition was approached. Later, Joslin, Streett and Chang (1993) and Berlin, Lundbladh and Henningson (1994) applied spatial numerical simulations covering the transition process to a Blasius boundary layer. A three stage process was observed for this transition scenario:(i) a nonlinear interaction of the primary disturbances $(f, \pm \beta)$ to generate a streamwise vortex system $(0,2 \beta)$,(ii) a transient growth region of low and high-speed streaks produced by the vortex system, and (iii) ultimate breakdown of the streaks instigated by non-stationary disturbances due to secondary instability.

The primary objectives of this research were to provide careful numerical experiments that can identify the controlling physics of the laminar-to-turbulent transition process, and to supply a potential database for comparison with theory and/or numerical simulations. This study is a logical extension of the work by Deng et al $(2004,2005)$ where two-dimensional and three-dimensional T-S disturbances were introduced into a Blasius boundary layer.

\subsection{Turbulence}

Since the beginning of the last century, many questions related to turbulence have been raised and a large number of empirical and heuristical results were derived and 
motivated by engineering applications. These includes the work of Lamb (1957), mostly on addressing idealized inviscid flows; Prandtl (1904), on eddy viscosity and boundary layers; Taylor (1935, 1937), on viscous flows; and von Karman (1911, 1912), on the nature of the boundary layer; the empirical skin friction coefficient of flat-plate turbulent boundary layer developed by Cousteix (1989).

In mechanical engineering, the interests are focus on certain physical quantities (forces, velocities, pressures, etc.). By considering these, another critical property of turbulence comes to mind: in a turbulent flow, many interesting quantities vary quickly and randomly in time and cannot be well measured. In practice, all that can be measured in laboratory experiments are mean values (usually time averaged). These averages are well-defined, reproducible quantities. This leads to the concept of ensemble averages underlying the conventional theory of turbulence, and to the concept of statistical solutions of the Navier-Stokes equations. The popular variables people are interested in are Reynolds stress, rms of the energy fluctuation and spectra analysis.

Turbulent flows seem to display self-similar statistical properties at length scales smaller than the scales at which energy is delivered to the flow. Kolmogorov argued that, at these scales, in three dimensions, the fluids display universal statistical features. Turbulent flow is conventionally visualized as a cascade of large eddies (large-scale components of the flow)breaking up successively into very smaller sized eddies. Such a cascade, or flow of kinetic energy from large to small scales, is taken to account in a regime at lengths sufficiently large for the effects of viscosity to be neglegible. The apparent energy dissipation - that is, the removal of energy from one length scale to a smaller one - is solely due to the presence of the nonlinear (inertial)term in the Navier-Stokes equations. The energy dissipation rate $\epsilon=\nu \kappa_{0}^{3}|\nabla u(x, t)|^{2}$ is assumed to be constant in space and time. A further essential assumption is that the cascade proceeds so that, at every length scale (or at every corresponding wavenumber), there is an equilibrium between energy 
flowing in from above to a given scale and that flowing out to a short scale. Such a picture and the associated assumptions imply that, in this range of length scales (or this range of wavenumbers), the energy density at a given wavenumber can depend only on the energy dissipation rate $\epsilon$ and the wavenumber $\mathrm{k}$ itself. Then dimensional analysis alone yields $S(\kappa)=$ constant $\times \epsilon^{2 / 3} / \kappa^{5 / 3}$ for the energy density. Such a cascade process cannot continue to arbitrarily small length scales because, as the norm of the Laplacian operator increases with the decreasing length scale, eventually the effects of molecular dissipation begin to dominate the nonlinear inertial term. That length, denoted by $l_{d}$, is the endpoint of the inertial range and the beginning of the dissipation range. Let us determine $\ell_{d}$. At each scale $\ell$ (or wavenumber $\kappa=\ell^{-1}$ ), we can define by dimensional analysis, through $\epsilon$ and $\ell$, a natural time scale $\tau$ and speed $\mathrm{u}$. Indeed, $\epsilon=\ell^{2} / \tau^{3}$ gives $\tau=\left(\ell^{2} / \epsilon\right)^{1 / 3}$ and $u=\ell / \tau=(\ell \epsilon)^{1 / 3}$. Now, the dissipation length $\ell_{d}$ is where the viscous term $\nu \nabla u$ starts to dominate, on average, the inertial term.

Hence,

$$
\nu \nabla u \sim \frac{\nu u}{\ell^{2}} \sim \frac{\nu}{\ell \tau}>(u \cdot \nabla) u \sim \frac{u^{2}}{\ell} \frac{\ell}{\tau^{2}}
$$

Therefore,

$$
\ell^{2}<\nu \tau=\nu \frac{\ell^{2}}{\epsilon} \Leftrightarrow \ell^{4 / 3}<\left(\frac{\nu^{3}}{\epsilon}\right)^{1 / 3}
$$

and

$$
\ell_{d}=\left(\frac{\nu^{3}}{\epsilon}\right)^{1 / 4}
$$

Kolmogorov thus inferred that, in three-dimensional turbulent flows, the eddies of length size sensibly smaller than $\ell_{d}$ are of no dynamical consequence. As we said, the length $\ell_{d}$ as defined by (3.1)is known as the Kolmogorov dissipation length. The corresponding wavenumber, $\kappa_{d}=\left(\frac{1}{\ell_{d}}\right)=\left(\frac{\epsilon}{\nu^{3}}\right)^{1 / 4}$ is the Kolmogorov dissipation wavenumber. 
The inertial range, within which inertial effects dominate, is the range $\ell_{1}<\ell<\ell_{d}$, where $\ell_{1}=L 1$ is the wavelength at which energy is injected in the flow. To each $\ell$ length in this range we can associate a Reynolds number $R e_{\ell}=u \ell / \nu$; hence,

$$
R e_{\ell}^{3 / 4}=\ell\left(\left(\frac{\epsilon}{\nu^{3}}\right)^{1 / 4}\right)
$$

The largest of these Reynolds numbers obtained for $\ell=$ the Kolmogorov macro-scale length $L_{\star} \approx \mathrm{E}_{1}$ is the Reynolds number Re of the flow. Hence,

$$
R e=\left(\frac{L_{\star}}{\ell_{d}}\right)^{4 / 3} \text { or } L_{\star}=R e^{3 / 4} \ell_{d}
$$

This relationship leads naturally to the heuristic estimate of the number of degrees of freedom in 3-dimensional flows, which is $R e^{9 / 4}$. As we shall see, this heuristic estimate is actually an upper bound on the sufficient (but not necessary)number of degrees of freedom in 3-dimensional turbulent flows. We now present a somewhat more elaborate derivation (but one that is still divorced from the Navier-Stokes equations) of the so-called Kolmogorov spectrum.

Let $\epsilon$ denote the average of the energy per unit mass. Then, according to the Kolmogorov theory, the length $\ell_{d}$ at which the turbulent eddies are rapidly annihilated by the viscosity should be a universal function of $\epsilon$ and the kinematic viscosity $\nu$,namely:

$$
\ell_{d}=f(\nu, \epsilon)
$$

In particular, f should be independent of the choice of units for space and time. Thus, if we pass from $\mathrm{x}$, t to $x^{\prime}=\xi x$ and $t^{\prime}=\tau t$ then we should still have

$$
\ell_{d}^{\prime}=f\left(\nu^{\prime}, \epsilon^{\prime}\right)
$$

Here $\nu^{\prime}$ and $\epsilon^{\prime}$ are not independent of $\nu$ and $\epsilon$, and dimensional analysis yields

$$
\ell_{d}^{\prime}=\xi \ell_{d}, \nu^{\prime}=\frac{\xi^{2}}{\tau} \nu, \epsilon^{\prime}=\frac{\xi^{2}}{\tau^{3}} \epsilon
$$


That is

$$
\xi f(\nu, \epsilon)=f\left(\xi^{2} \tau^{-1} \nu, \xi^{2} \tau^{-3} \epsilon\right)
$$

With the choice

$$
\frac{\xi^{2}}{\tau}=\frac{1}{\nu} \text { and } \frac{\xi^{2}}{\tau^{3}}=\frac{1}{\epsilon},\left(\text { i.e. } \tau=\left(\epsilon \nu^{1 / 2}\right) \text { and } \xi=\epsilon^{1 / 4} / \nu^{3 / 4}\right)
$$




\section{CHAPTER 3}

\section{GOVERNING EQUATIONS AND NUMERICAL METHODS}

\subsection{Governing Equations}

The three-dimensional compressible Navier-Stokes equations in generalized curvilinear coordinates $(\xi, \eta, \zeta)$ can be written in conservative forms:

$$
\frac{1}{J} \frac{\partial Q}{\partial t}+\frac{\partial\left(E-E_{v}\right)}{\partial \xi}+\frac{\partial\left(F-F_{v}\right)}{\partial \eta}+\frac{\partial\left(G-G_{v}\right)}{\partial \zeta}=0
$$

The vector of conserved quantities $Q$, inviscid flux vector $(E, F, G)$, and viscous flux vector $\left(E_{v}, F_{v}, G_{v}\right)$ are defined via

$$
\begin{gathered}
Q=\left(\begin{array}{c}
\rho \\
\rho u \\
\rho v \\
\rho w \\
e
\end{array}\right) \quad E=\frac{1}{J}\left(\begin{array}{c}
\rho U \\
\rho U u+p \xi_{x} \\
\rho U v+p \xi_{y} \\
\rho U w+p \xi_{z} \\
U(e+p)
\end{array}\right) \quad F=\frac{1}{J}\left(\begin{array}{c}
\rho V \\
\rho V u+p \eta_{x} \\
\rho V v+p \eta_{y} \\
\rho V w+p \eta_{z} \\
\rho W(e+p)
\end{array}\right) \\
G=\frac{1}{J}\left(\begin{array}{c} 
\\
\rho W v+p \zeta_{y} \\
\rho W w+p \zeta_{z} \\
W(e+p) \\
E_{v}=\frac{1}{J} \\
0 \\
\tau_{x x} \xi_{x}+\tau_{y x} \xi_{y}+\tau_{z x} \xi_{z}+\tau_{y y} \xi_{y}+\tau_{z y} \xi_{z} \\
\tau_{x z} \xi_{x}+\tau_{y z} \xi_{y}+\tau_{z z} \xi_{z} \\
q_{x} \xi_{x}+q_{y} \xi_{y}+q_{z} \xi_{z}
\end{array}\right)
\end{gathered}
$$




$$
F_{v}=\frac{1}{J}\left(\begin{array}{c}
0 \\
\tau_{x x} \eta_{x}+\tau_{y x} \eta_{y}+\tau_{z x} \eta_{z} \\
\tau_{x y} \eta_{x}+\tau_{y y} \eta_{y}+\tau_{z y} \eta_{z} \\
\tau_{x z} \eta_{x}+\tau_{y z} \eta_{y}+\tau_{z z} \eta_{z} \\
q_{x} \eta_{x}+q_{y} \eta_{y}+q_{z} \eta_{z}
\end{array}\right) \quad G_{v}=\frac{1}{J}\left(\begin{array}{c}
0 \\
\tau_{x x} \zeta_{x}+\tau_{y x} \zeta_{y}+\tau_{z x} \zeta_{z} \\
\tau_{x y} \zeta_{x}+\tau_{y y} \zeta_{y}+\tau_{z y} \zeta_{z} \\
\tau_{x z} \zeta_{x}+\tau_{y z} \zeta_{y}+\tau_{z z} \zeta_{z} \\
q_{x} \zeta_{x}+q_{y} \zeta_{y}+q_{z} \zeta_{z}
\end{array}\right),
$$

where $J \equiv \frac{\partial(\xi, \eta, \zeta)}{\partial(x, y, z)}$ is Jacobian of the coordinate transformation between the curvilinear $(\xi, \eta, \zeta)$ and cartesian $(x, y, z)$ frames, and $\xi_{x}, \xi_{y}, \xi_{z}, \eta_{x}, \eta_{y}, \eta_{z}, \zeta_{x}, \zeta_{y}, \zeta_{z}$ are coordinate transformation metrics. The contravariant velocity components $U, V, W$ are defined as

$$
U=u \xi_{x}+v \xi_{y}+w \xi_{z}, \quad V=u \eta_{x}+v \eta_{y}+w \eta_{z}, \quad W=u \zeta_{x}+v \zeta_{y}+w \zeta_{z}
$$

where $e$ denotes the total energy,

$$
e=\frac{p}{\gamma-1}+\frac{1}{2} \rho\left(u^{2}+v^{2}+w^{2}\right)
$$

The components of the viscous stress tensor and heat flux are denoted by $\tau_{x x}, \tau_{y y}, \tau_{z z}$, $\tau_{x y}, \tau_{x z}, \tau_{y z}$, and $q_{x}, q_{y}, q_{z}$, respectively.

In the dimensionless form, the reference values for length, density, velocities, temperature, pressure and time are $\delta_{i n}, \rho_{\infty}, U_{\infty}, T_{\infty}, \rho_{\infty} U_{\infty}^{2}$, and $\frac{\delta_{i n}}{U_{\infty}}$ respectively, where $\delta_{i n}$ denotes the displacement thickness at the inflow boundary. The Mach number $M_{\infty}$, Reynolds number Re, Prandtl number $P r$, and the ratio of specific heats $\gamma$, are defined respectively as follows:

$$
M_{\infty}=\frac{U_{\infty}}{\sqrt{\gamma R T_{\infty}}}, \quad R e=\frac{\rho_{\infty} U_{\infty} \delta_{i n}}{\mu_{\infty}}, \quad \operatorname{Pr}=\frac{C_{p} \mu_{\infty}}{\kappa_{\infty}}, \quad \gamma=\frac{C_{p}}{C_{v}}
$$

where $R$ is the ideal gas constant, $C_{p}$ and $C_{v}$ are specific heats at constant pressure and constant volume, respectively. Through out this work, $\operatorname{Pr}=0.7$ and $\gamma=1.4$. Viscosity is determined according to the Sutherland's law in dimensionless form

$$
\mu=\frac{T^{3 / 2}(1+S)}{T+S}, \quad S=\frac{110.3 K}{T_{\infty}} .
$$


Eq. (3.1) can also be written as

$$
\frac{\partial Q}{\partial t}=R
$$

where the right hand side $R$ is given by

$$
R=-J\left[\left(D_{\xi}\left(E-E_{v}\right)+D_{\eta}\left(F-F_{v}\right)+D_{\zeta}\left(G-G_{v}\right)\right]\right.
$$

$D_{\xi}, D_{\eta}, D_{\zeta}$ being partial differential operators in $\xi, \eta$, and $\zeta$ directions, respectively.

\subsection{Numerical Methods}

The right hand side of Eq. (3.4) is discretized using the sixth-order compact scheme (Lele, 1992) for spatial derivatives in the streamwise $(\xi)$, spanwise $(\eta)$, and wall-normal $(\zeta)$ directions. In the spanwise direction, the spectral method can also be used in the place of the compact scheme. For internal points $j=3, \ldots, N-2$, the sixth order compact scheme is as follows

$$
\frac{1}{3} f_{j-1}^{\prime}+f_{j}^{\prime}+\frac{1}{3} f_{j+1}^{\prime}=\frac{1}{h}\left(-\frac{1}{36} f_{j-2}-\frac{7}{9} f_{j-1}+\frac{7}{9} f_{j+1}+\frac{1}{36} f_{j+2}\right),
$$

where $f_{j}^{\prime}$ is the derivative at point $j$. The fourth order compact scheme is used at points $j=2, N-1$, and the third order one-sided compact scheme is used at the boundary points $j=1, N$

In order to eliminate the spurious numerical oscillations caused by central difference schemes, a high-order spatial is used instead of artificial dissipation. Implicit sixthorder compact scheme for space filtering (Lele, 1992) is applied for primitive variables $u, v, w, \rho, p$ after a specified number of time steps. 
In Eq. (3.4), a third-order TVD Runge-Kutta method (Shu, 1988) is used for time-integration:

$$
\begin{aligned}
Q^{(0)} & =Q^{n} \\
Q^{(1)} & =Q^{(0)}+\Delta t R^{(0)} \\
Q^{(2)} & =\frac{3}{4} Q^{(0)}+\frac{1}{4} Q^{(1)}+\frac{1}{4} \Delta t R^{(1)} \\
Q^{n+1} & =\frac{1}{3} Q^{(0)}+\frac{2}{3} Q^{(2)}+\frac{2}{3} \Delta t R^{(2)}
\end{aligned}
$$

$C F L \leq 1$ is required to ensure the stability. The stability constraint restrict the efficiency when applying explicit schemes to flows with low Mach number $\left(M_{\infty} \leq 0.3\right)$. Flows with Mach number around 0.15 can be considered as incompressible flows. For flow solver, implicit scheme or explicit scheme with preconditioning is recommended.

\subsection{Boundary Conditions}

The following boundary conditions are used during the preliminary validation exercises described in this report.

1. Wall boundary condition:

- Non-slip condition for velocity:

$$
u=v=w=0
$$

- Adiabatic condition for temperature:

$$
\frac{\partial T}{\partial n}=0
$$

The second order scheme is used to approximate the temperature derivative.

$$
T_{1}=\left(4 T_{2}-T_{3}\right) / 3
$$


- Boundary condition for pressure:

$$
\frac{\partial p}{\partial n}=0
$$

The second order discrete form is

$$
p_{1}=\left(4 p_{2}-p_{3}\right) / 3
$$

2. Upstream boundary condition:

$$
q=q_{l a m}+A_{2 d} q_{2 d}^{\prime}+A_{3 d} q_{3 d}^{\prime}+\text { Noise }
$$

where $q$ stands for velocity components, pressure, and density. $q_{\text {lam }}$ is the two-dimensional Blasius-like profile obtained from solving the similarity equation. $q_{2 d}^{\prime}$ and $q_{3 d}^{\prime}$ are the twodimensional and three-dimensional forcing. $A_{2 d}$ and $A_{3 d}$ are the amplitudes of perturbations. Because the inflow is subsonic, only four boundary conditions are needed here. But for the test cases described in this report, the inlet flow is given by the similarity solution of NS equations and the amplitudes of perturbations are very small, so the over specification of boundary conditions here didn't cause problem in our computations. According to our experience, if the physical values imposed at the inlet are not the solution of the equations or the perturbations are not small enough, some sort of characteristic boundary conditions should be applied here.

3. Non-reflecting boundary condition(Jiang, 1999) 
Non-reflecting boundary condition proposed in (Jiang,1999b) are used at the boundaries of far field and outflow. Non-reflecting boundary condition for inflow is also included in the code. Based on the characteristic analysis, Eq. (3.1) can be rewritten as

$$
\begin{array}{r}
\frac{\partial \rho}{\partial t}+d_{1}+V \frac{\partial \rho}{\partial \eta}+\rho\left(\eta_{x} \frac{\partial u}{\partial \eta}+\eta_{y} \frac{\partial v}{\partial \eta}+\eta_{z} \frac{\partial w}{\partial \eta}\right)+W \frac{\partial \rho}{\partial \zeta} \\
+\rho\left(\zeta_{x} \frac{\partial u}{\partial \zeta}+\zeta_{y} \frac{\partial v}{\partial \zeta}+\zeta_{z} \frac{\partial w}{\partial \zeta}\right)+v i s_{1}=0 \\
\frac{\partial u}{\partial t}+d_{2}+V \frac{\partial u}{\partial \eta}+\frac{1}{\rho} \eta_{x} \frac{\partial p}{\partial \eta}+W \frac{\partial u}{\partial \zeta}+\frac{1}{\rho} \zeta_{x} \frac{\partial p}{\partial \zeta}+v i s_{2}=0 \\
\frac{\partial v}{\partial t}+d_{3}+V \frac{\partial v}{\partial \eta}+\frac{1}{\rho} \eta_{y} \frac{\partial p}{\partial \eta}+W \frac{\partial v}{\partial \zeta}+\frac{1}{\rho} \zeta_{y} \frac{\partial p}{\partial \zeta}+v i s_{3}=0 \\
\frac{\partial w}{\partial t}+d_{4}+V \frac{\partial w}{\partial \eta}+\frac{1}{\rho} \eta_{z} \frac{\partial p}{\partial \eta}+W \frac{\partial w}{\partial \zeta}+\frac{1}{\rho} \zeta_{z} \frac{\partial p}{\partial \zeta}+v i s_{4}=0 \\
\frac{\partial p}{\partial t}+d_{5}+V \frac{\partial p}{\partial \eta}+\gamma p\left(\eta_{x} \frac{\partial u}{\partial \eta}+\eta_{y} \frac{\partial v}{\partial \eta}+\eta_{z} \frac{\partial w}{\partial \eta}\right)+W \frac{\partial p}{\partial \zeta} \\
+\gamma p\left(\zeta_{x} \frac{\partial u}{\partial \zeta}+\zeta_{y} \frac{\partial v}{\partial \zeta}+\zeta_{z} \frac{\partial w}{\partial \zeta}\right)+v i s_{5}=0
\end{array}
$$

where vector $\boldsymbol{d}$ is obtained from the characteristic analysis,

$$
\left(\begin{array}{l}
d_{1} \\
d_{2} \\
d_{3} \\
d_{4} \\
d_{5}
\end{array}\right)=\left(\begin{array}{c}
\frac{1}{c^{2}}\left[\frac{1}{2}\left(\mathcal{L}_{1}+\mathcal{L}_{5}\right)+\mathcal{L}_{2}\right] \\
\frac{\xi_{x}}{2 \beta \rho c}\left(\mathcal{L}_{5}-\mathcal{L}_{1}\right)-\frac{1}{\beta^{2}}\left(\xi_{y} \mathcal{L}_{3}+\xi_{z} \mathcal{L}_{4}\right) \\
\frac{\xi_{y}}{2 \beta \rho c}\left(\mathcal{L}_{5}-\mathcal{L}_{1}\right)+\frac{1}{\beta^{2} \xi_{x}}\left[\left(\xi_{x}^{2}+\xi_{z}^{2}\right) \mathcal{L}_{3}-\xi_{z} \xi_{y} \mathcal{L}_{4}\right] \\
\frac{\xi_{z}}{2 \beta \rho c}\left(\mathcal{L}_{5}-\mathcal{L}_{1}\right)-\frac{1}{\beta^{2} \xi_{x}}\left[\xi_{y} \xi_{z} \mathcal{L}_{3}-\left(\xi_{x}^{2}+\xi_{y}^{2}\right) \mathcal{L}_{4}\right] \\
\frac{1}{2}\left(\mathcal{L}_{1}+\mathcal{L}_{5}\right)
\end{array}\right)
$$

where $c$ is the sound speed, and

$$
\beta=\sqrt{\xi_{x}^{2}+\xi_{y}^{2}+\xi_{z}^{2}}
$$

$\mathcal{L}_{i}$ 's represent the amplitude variations of the characteristic waves corresponding to the characteristic velocities $\lambda_{i}$ 's, which are given by

$$
\begin{array}{r}
\lambda_{1}=U-C_{\xi} \\
\lambda_{2}=\lambda_{3}=\lambda_{4}=U \\
\lambda_{5}=U+C_{\xi},
\end{array}
$$


where $C_{\xi}=c \beta$, and $\mathcal{L}_{i}$ 's can be expressed as

$$
\begin{aligned}
\mathcal{L}_{1} & =\left(U-C_{\xi}\right)\left[-\frac{\rho c}{\beta}\left(\xi_{x} \frac{\partial u}{\partial \xi}+\xi_{y} \frac{\partial v}{\partial \xi}+\xi_{z} \frac{\partial w}{\partial \xi}\right)+\frac{\partial p}{\partial \xi}\right] \\
\mathcal{L}_{2} & =U\left(c^{2} \frac{\partial \rho}{\partial \xi}-\frac{\partial p}{\partial \xi}\right) \\
\mathcal{L}_{3} & =U\left(-\xi_{y} \frac{\partial u}{\partial \xi}+\xi_{x} \frac{\partial v}{\partial \xi}\right) \\
\mathcal{L}_{4} & =U\left(-\xi_{z} \frac{\partial u}{\partial \xi}+\xi_{x} \frac{\partial w}{\partial \xi}\right) \\
\mathcal{L}_{5} & =\left(U+C_{\xi}\right)\left[\frac{\rho c}{\beta}\left(\xi_{x} \frac{\partial u}{\partial \xi}+\xi_{y} \frac{\partial v}{\partial \xi}+\xi_{z} \frac{\partial w}{\partial \xi}\right)+\frac{\partial p}{\partial \xi}\right]
\end{aligned}
$$

The terms vis ${ }_{i}$ 's in Eq. (3.6) represent the viscous terms. The analogous definitions can be made for the other two directions.

- Outflow: For subsonic outflow at $\xi=N_{x}$, four characteristic waves $\mathcal{L}_{2}, \mathcal{L}_{3}, \mathcal{L}_{4}, \mathcal{L}_{5}$ are going out of the computational domain, while $\mathcal{L}_{1}$ is entering the field. Therefore, $\mathcal{L}_{2}, \mathcal{L}_{3}, \mathcal{L}_{4}, \mathcal{L}_{5}$ can be calculated from the interior points using Eq. (3.12) with the compact finite difference scheme, while the $\mathcal{L}_{1}$ is set to zero.

- Far Field: For far field boundary at $\zeta=N_{z}$, the directions of characteristic waves are determined automatically by local field values, and $\mathcal{L}_{i}$ of the outgoing waves are calculated from the interior points using Eq. (3.12) in $\zeta$ direction. Those inward going waves are set to zero. i.e.

$$
\mathcal{L}_{i}=\left\{\begin{array}{cc}
\mathcal{L}_{i}, & \text { for } \lambda_{i}>0 \\
0, & \text { for } \lambda_{i}<0
\end{array}\right.
$$

- Inflow: For subsonic inflow at $\xi=1$, four quantities should be specified, i.e. $u, v, w, T$, while the density $\rho$ is obtained by solving Eq. (3.6). This arrangement is made based on the fact that the four characteristic waves $\mathcal{L}_{2}, \mathcal{L}_{3}, \mathcal{L}_{4}, \mathcal{L}_{5}$ are entering the computational domain, while $\mathcal{L}_{1}$ is going outward. Therefore, $\mathcal{L}_{1}$ is calculated from interior points using Eq. (3.12), where the spatial derivatives are 
calculated using the compact finite difference scheme, and $\mathcal{L}_{2}, \mathcal{L}_{3}, \mathcal{L}_{4}, \mathcal{L}_{5}$ are given in $(3.14)-(3.17)$.

$$
\begin{aligned}
\mathcal{L}_{3} & =\xi_{y} \frac{\partial u}{\partial t}-\xi_{x} \frac{\partial v}{\partial t} \\
\mathcal{L}_{4} & =\xi_{z} \frac{\partial u}{\partial t}-\xi_{x} \frac{\partial w}{\partial t} \\
\mathcal{L}_{5} & =\mathcal{L}_{1}+\frac{2 \beta \rho c}{\xi_{x}}\left[\frac{1}{\beta^{2}}\left(\xi_{y} \mathcal{L}_{3}+\xi_{z} \mathcal{L}_{4}\right)-\frac{\partial u}{\partial t}\right] \\
\mathcal{L}_{2} & =\frac{\rho}{M_{r}^{2}} \frac{\partial T}{\partial t}+\frac{1}{2}\left(\mathcal{L}_{1}+\mathcal{L}_{5}\right)
\end{aligned}
$$

5. Spanwise boundary condition:

- The periodic condition

Compact finite difference scheme and spectral method are available in the code to calculate the derivative in the spanwise direction. The period condition is implicitly imposed as $q_{1}=q_{N_{y}+1}$.

\subsection{Parallel Computing}

The parallel version of the numerical simulation code based on the Message Passing Interface (MPI) has been developed to improve the performance. The parallel computing is combined with domain decomposition method. The computational domain is divided into $n$ equal-sized subdomains along the $\xi$ direction as shown in Figure 3.1, where $n$ is the number of processors. This is a simple partition with a balanced load for each processor. During computation, a processor communicates with it neighbors through exchanging the data at left and right boundary of each subdomain. But this type of communication is not suitable for calculating derivative in the $\xi$ direction while using the compact finite difference scheme. If each grid node along a $\xi$ grid line locates in the same processor, it will be straightforward to use the compact scheme. In Figure 3.2, a data structure with four processors is used as an example to illustrate a special type of 
data exchange which has been utilized to accomplish the data structure transformation. The figure on the top shows the original partition where the computational domain is divided along the $\xi$ direction. This data structure can be transformed to a new structure shown in the figure on the bottom where the domain is divided along the $\zeta$ direction. The transformation is accomplished by first defining two new data types and then calling a MPI routine "MPI_ALLTOALL" from the MPI library. In the new data structure, all the grid nodes along a $\xi$ grid line are stored in one processor. After the calculation of derivative is completed, an inverse transformation is used to recover the data structure back to the original one.



Figure 3.1 The domain decomposition along $\xi$ direction

This parallel version of the code is based on domain decomposition. The computational domain are evenly divided along the streamwise direction, so that each processor has the same load. To run sequential simulations, we can use one processor. 


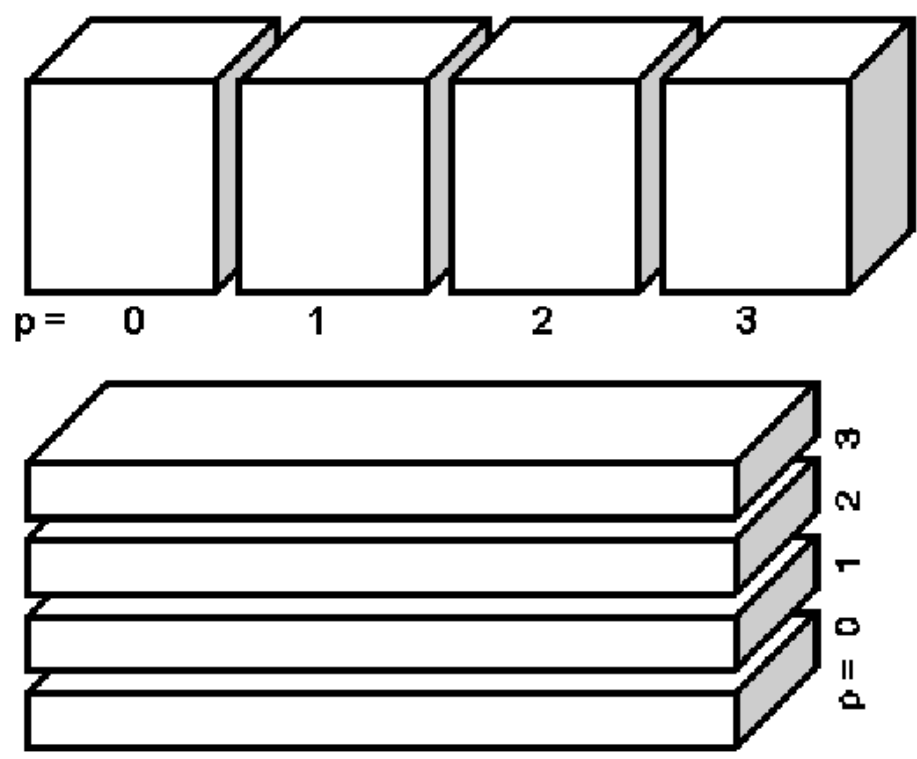

Figure 3.2 The change of data structure for calculating derivative in $\xi$ direction 


\section{CHAPTER 4}

\section{COMPUTATIONAL PROCEDURE AND RESULTS DISCUSSION}

\subsection{Computational Procedure}

The computation domain is displayed in Fig.4.1. Computation is carried out in three different grid levels: 256x32x64, 640x64x64 and 1536x128x64. For example, $256 \times 32 \times 64$ has 256 nodes in the streamwise, 32 in the spanwise and 64 in the wall normal directions. The length of computational domain along the streamwise direction is approximately 800 , the width along the spanwise direction is around 22 , and height at the inflow boundary is about 40. For different cases, the domain varies a little.



Figure 4.1 Computation domain

The flow parameters used throughout this work are listed in the Table 4.1. In the simulation, the Reynolds number is 1000 at the inflow boundary. The reference values for Reynolds number are based on the displacement thickness and the free-stream velocity. The March number is 0.5 . 
Table 4.1 Flow parameters

\begin{tabular}{cc}
\hline \hline$M_{\infty}$ & 0.5 \\
$R e_{i n}$ & 1000 \\
$P r$ & 0.7 \\
$X_{\text {in }}$ & $300.79 \delta_{\text {in }}$ \\
$T_{\infty}$ & 273 \\
\hline \hline
\end{tabular}

The inflow is given in the form of

$$
q=q_{l a m}+A_{2 d} q_{2 d}^{\prime}+A_{3 d} q_{3 d}^{\prime}+A_{r} q_{r}^{\prime}
$$

where $\mathrm{q}$ stands for the velocity components, the pressure, and the density. $q_{\text {lam }}$ is the two-dimensional Blasius-like profile obtained from the solution of the similarity equation. In Equation 4.1, $q_{2 d}^{\prime}$ represents the eigenmode of two-dimensional TollmienSchlichting (T-S) waves with a space wave number of $\alpha_{2 d}=\alpha_{2 d}^{r}+i \alpha_{2 d}^{i}$ and a frequency of $\omega_{2 d}$, it can be expressed as follows:

$$
q_{2 d}^{\prime}=\phi_{2 d}^{r} \cos \left(\omega_{2 d} t\right)+\phi_{2 d}^{i} \sin \left(\omega_{2 d} t\right)
$$

where $\phi_{2 d}^{r}$ and $\phi_{2 d}^{i}$ are the real and imaginary part of the two-dimensional eigenmode. $q_{3 d}^{\prime}$ in Equation 4.1 denotes the eigenmode of three-dimensional T-S waves with space wave numbers $\alpha_{3 d}=\alpha_{3 d}^{r}+i \alpha_{3 d}^{i}$ and $\beta$, and a frequency of $\omega_{3 d}$, in the following form:

$$
q_{3 d}^{\prime}=\phi_{3 d}^{r} \cos (\beta y) \cos \left(\omega_{3 d} t\right)+\phi_{3 d}^{r} \sin (\beta y) \sin \left(\omega_{3 d} t\right)-\phi_{3 d}^{i} \sin (\beta y) \cos \left(\omega_{3 d} t\right)+\phi_{3 d}^{i} \cos (\beta y) \sin \left(\omega_{3 d} t\right)
$$

where $\phi_{3 d}^{r}$ and $\phi_{3 d}^{i}$ are the real and imaginary part of the eigenmode of threedimensional disturbance. The disturbance enjoys the scaled shape of a three-dimensional T-S waves associated with a random white noise ranging from -1 to 1 is denoted by 
$q_{r}^{\prime}$ in Equation 4.1. $A_{2 d}, A_{3 d}$ and $A_{r}$ are the amplitude of the two-dimensional, threedimensional, and random noise, respectively.

To study K-type and H-type transition over a flat plate, this paper presents several simulation cases for different grids level: they distinguished by different inflow disturbance. The inflow consists of a two-dimensional T-S mode and a three-dimensional T-S mode on top of which a random noise is imposed. Naturally, for K-type transition, the perturbation amplitude is larger and the perturbation amplitude for $\mathrm{H}$-type is relatively smaller. The typical parameters used for the two transition types are shown in Table 4.2.

Table 4.2 Typical inflow disturbance parameters

\begin{tabular}{ccc}
\hline \hline & K-type & H-type \\
\hline$\alpha_{2 d}^{r}$ & 0.247 & 0.247 \\
$\alpha_{2 d}^{i}$ & $-6.611 \times 10^{-3}$ & $-6.611 \times 10^{-3}$ \\
$\omega_{2 d}$ & 0.0909 & 0.0909 \\
$A_{2 d}$ & 0.02 & 0.01 \\
$\alpha_{2 d}^{r}$ & 0.247 & 0.247 \\
$\alpha_{2 d}^{i}$ & $-6.611 \times 10^{-3}$ & $-6.611 \times 10^{-3}$ \\
$\omega_{2 d}$ & 0.0909 & 0.0909 \\
$A_{2 d}$ & 0.02 & 0.01 \\
$A_{r}$ & 0.01 & 0.005 \\
$\beta$ & 0.2 & 0.2 \\
\hline \hline
\end{tabular}

\subsection{Computational Results}

\subsubsection{Coarsest grids $(256 \times 32 \times 64)$}

\subsubsection{Case 1 - Without three dimensional TS wave}

This case ran with the chosen parameters listed in table 4.3. 
Table 4.3 Case 1 inflow parameters of coarsest grids

\begin{tabular}{cc}
\hline \hline & H-type \\
\hline$\alpha_{2 d}^{r}$ & 0.312 \\
$\alpha_{2 d}^{i}$ & $-3.837 \times 10^{-3}$ \\
$\omega_{2 d}$ & 0.12 \\
$A_{2 d}$ & 0.005 \\
$\alpha_{3 d}^{r}$ & - \\
$\alpha_{3 d}^{i}$ & - \\
$\omega_{3 d}$ & - \\
$A_{3 d}$ & - \\
$A_{r}$ & 0.008 \\
$\beta$ & 0.0 \\
\hline \hline
\end{tabular}

From the instantaneous flow field in Fig 4.2, no transition is observed.

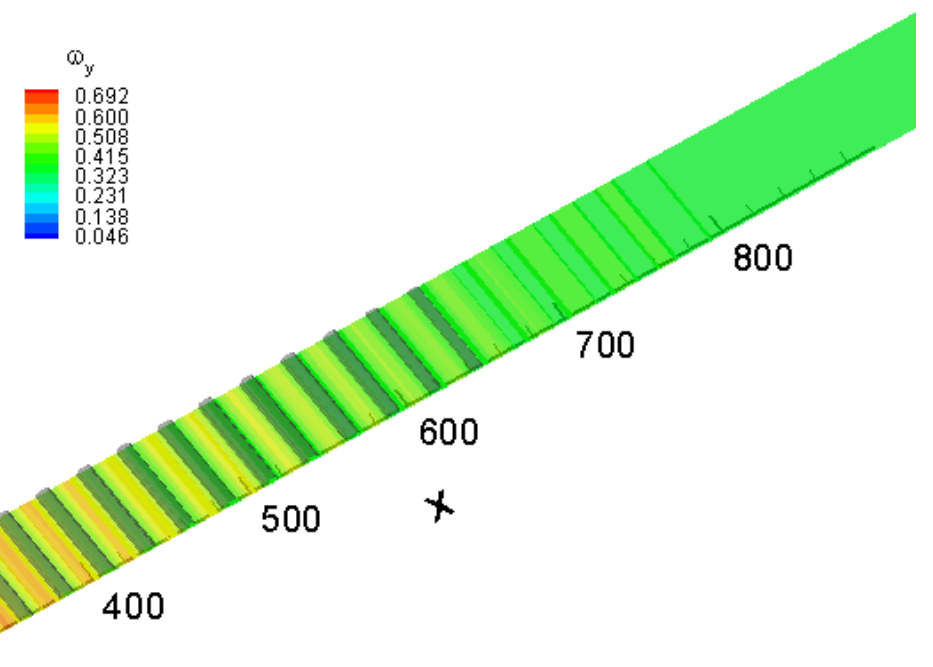

Figure 4.2 Case 1 instantaneous flow field 


\subsubsection{Case 2 - With three dimensional TS wave}

This case ran with the chosen parameters listed in table 4.4.

Table 4.4 Case 2 inflow parameters of coarsest grids

\begin{tabular}{cc}
\hline \hline & H-type \\
\hline$\alpha_{2 d}^{r}$ & 0.247 \\
$\alpha_{2 d}^{i}$ & $-6.611 \times 10^{-3}$ \\
$\omega_{2 d}$ & 0.0909 \\
$A_{2 d}$ & 0.005 \\
$\alpha_{3 d}^{r}$ & 0.247 \\
$\alpha_{3 d}^{i}$ & $-6.611 \times 10^{-3}$ \\
$\omega_{3 d}$ & 0.0909 \\
$A_{3 d}$ & 0.004 \\
$A_{r}$ & 0.002 \\
$\beta$ & 0.2 \\
\hline \hline
\end{tabular}

After the solution is well established in the whole domain, the spatial evolutions of the Fourier modes are studied. During one forcing period, the Fourier transform is carried out in time and spanwise direction to obtain the spectral components $\left(k_{t}, k_{y}\right)$ in the Fourier space, where $k_{t}$ refers the temporal wavenumber, and $k_{y}$ denotes the spanwise wavenumber. The mean flow is characterized by the spectral component $(0,0)$. The maximum amplitudes of streamwise velocity perturbation of selected Fourier components are plotted as a function of $\mathrm{x}$ in Figure 4.3. The Fourier component $(1,0)$ is corresponding to the imposed two-dimensional TS wave disturbance at the inflow boundary. The Fourier component $(1,1)$ is corresponding to the imposed three-dimensional TS wave disturbance at the inflow boundary. 


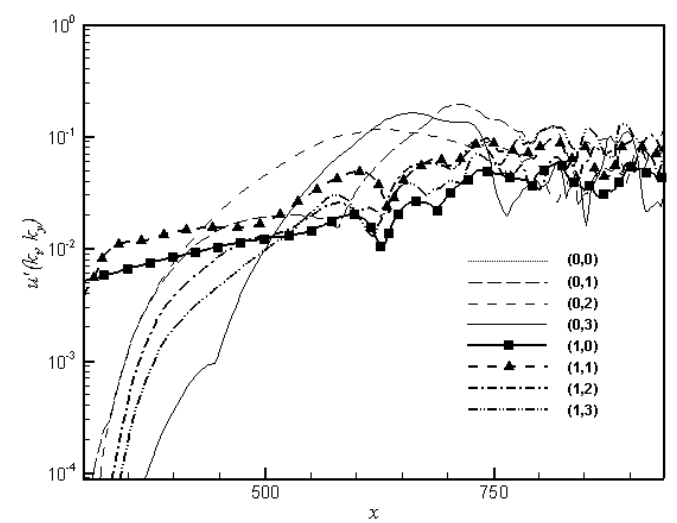

Figure 4.3 Case 2 spatial growth of streamwise perturbation

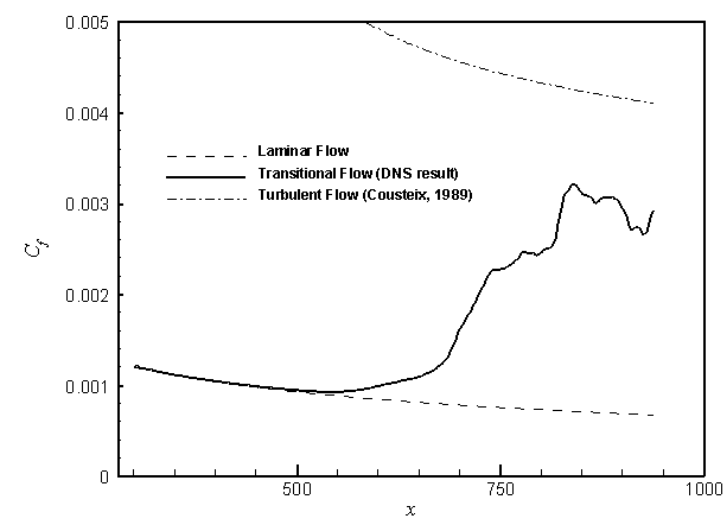

Figure 4.4 Case 2 skin friction comparison

From Figure 4.4 clear transition is observed. Compare to the first case, we have a same two dimensional TS wave, smaller noise amplitude and a additional three dimensional disturbance. In this case the extra three dimensional disturbance gives us the transition. For this case, the inflow perturbation is relatively low, H-type transition is expected.

From the instantaneous flow field in Figure 4.5, we can see the $\Lambda$ shape vortical structures appear at $x=500 \delta_{i n}$ and has a aligned pattern up to $x=700 \delta_{i n}$. Comparing 


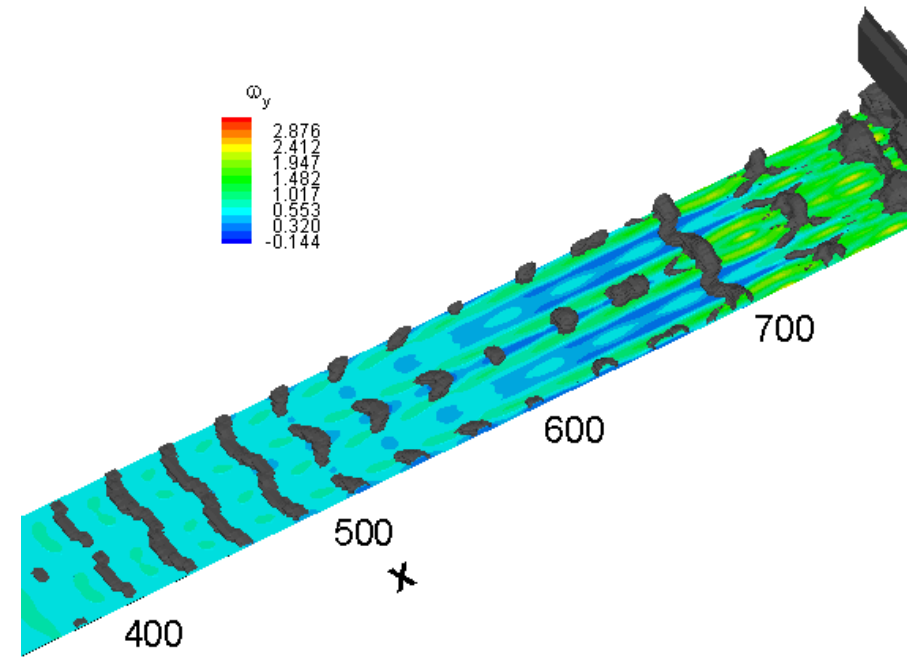

Figure 4.5 Case 2 instantaneous flow field

with Figure 4.4, transition starts around $x=700 \delta_{\text {in }}$ At that location, the flow tries to excite the subharmonic mode which is staggered pattern. As in the inflow, all the TS waves $(2 \mathrm{D}, 3 \mathrm{D})$ are harmonic modes, it is somewhat difficult to excite subharmonic mode with such a low noise level in so coarse grids. But we do observe subharmonic mode got excited with a noise level of $0.2 \%$. That we consider a H-type transition under low inflow perturbation.

\subsubsection{Case 3 - Two 3D TS wave with different $\omega$}

To see a clearer H-type transition, in case 3 both harmonic and subharmonic modes are imposed, refer to Table 4.5. In lower level perturbation than the harmonic modes, the subharmonic mode is picked up, got excited and dominated the behavior of transition flow.

As previous case, after the solution is well established in the whole domain, the spatial evolutions of the Fourier modes are studied and plotted in Figure 4.6. The Fourier 
Table 4.5 Case 3 inflow parameters of coarsest grids

\begin{tabular}{cc}
\hline \hline & H-type \\
\hline$\alpha_{2 d}^{r}$ & 0.312 \\
$\alpha_{2 d}^{i}$ & $-3.837 \times 10^{-3}$ \\
$\omega_{2 d}$ & 0.12 \\
$A_{2 d}$ & 0.005 \\
$\alpha_{3 d 1}^{r}$ & 0.312 \\
$\alpha_{3 d 1}^{i}$ & $-3.837 \times 10^{-3}$ \\
$\omega_{3 d 1}$ & 0.12 \\
$A_{3 d 1}$ & 0.004 \\
$\alpha_{3 d 2}^{r}$ & 0.155 \\
$\alpha_{3 d 2}^{i}$ & $-7.344 \times 10^{-4}$ \\
$\omega_{3 d 2}$ & 0.06 \\
$A_{3 d 2}$ & 0.002 \\
$A_{r}$ & 0.002 \\
$\beta$ & 0.2 \\
\hline \hline
\end{tabular}

component $(1,0)$ is corresponding to the imposed two-dimensional TS wave disturbance at the inflow boundary with $\omega=0.12$. The Fourier component $(1,1)$ is corresponding to the imposed harmonic three-dimensional TS wave disturbance at the inflow boundary with same temporal wave number as the two-dimensional TS wave. Here $(0,1)$ is the Fourier component corresponding to the imposed subharmonic three-dimensional TS wave disturbance at the inflow boundary with half the temporal wave number of the two-dimensional TS wave $\omega=0.06$.

From Figure 4.7 transition is observed. The post transition skin friction is a little low compare with case 2 . One possible answer is that the domain is smaller in this case, didn't allow the disturbance to grow further. The reason for such a small domain is to save some computational time. As in such coarse grid level, to study statistics about the flow field is not realistic. But even for coarse grid level and limited domain, the 


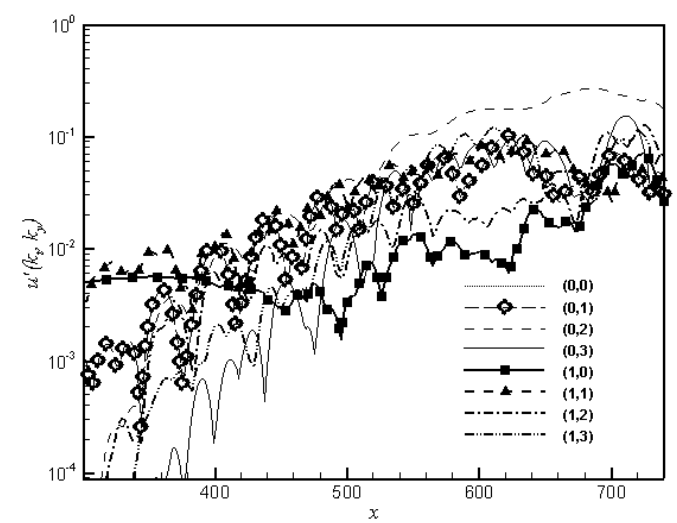

Figure 4.6 Case 3 spatial growth of streamwise perturbation

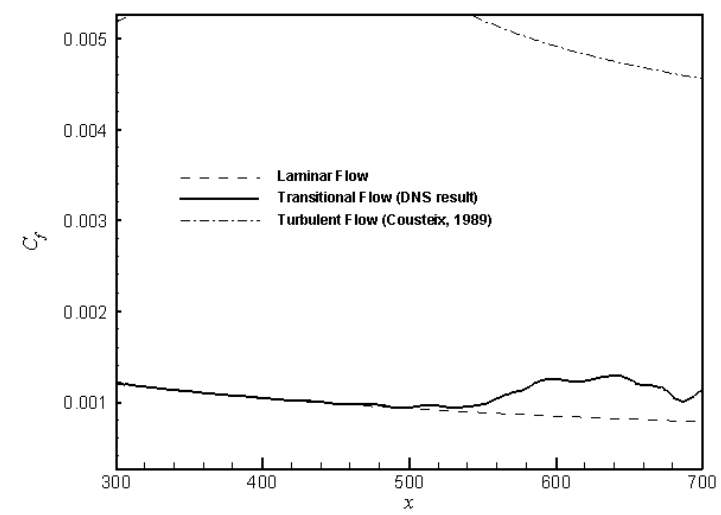

Figure 4.7 Case 3 skin friction comparison

flow behavior and the overall vortex structure is very close to what is happening in real world. We got enough information and the results is in good agreement with secondary instability theory and other people's numerical results.

For this case, the inflow perturbation is again relatively low, H-type transition is expected. From instantaneous flow field in Figure 4.8, a clear staggered pattern in the transition zone is observe. The $\Lambda$ shape vortical structures appear at $x=420 \delta_{\text {in }}$ and have a staggered pattern afterward. This indicates a H-type transition. 


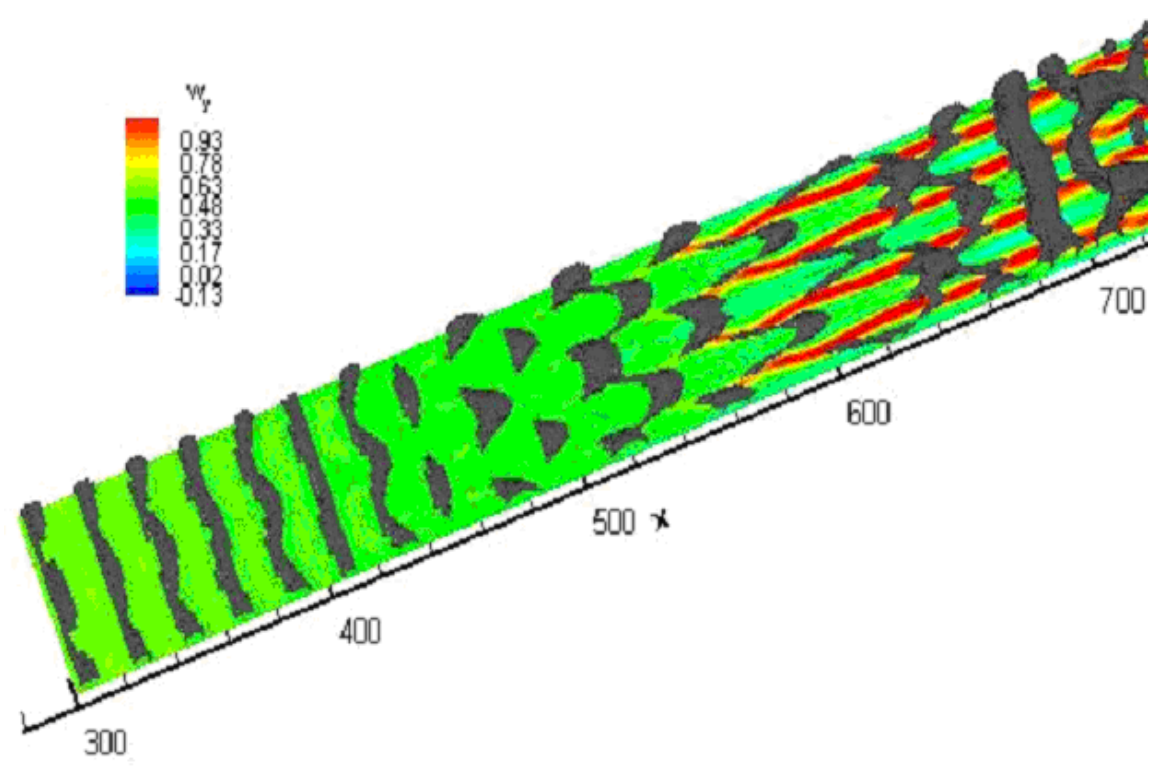

Figure 4.8 Case 3 instantaneous flow field

\subsubsection{Fine grids $(640 \times 64 \times 64)$}

\subsubsection{Case $4 \mathrm{~K}$-type transition in finer grids}

This case ran with the chosen parameters listed in table 4.6.

As previous case, after the solution is well established in the whole domain, the spatial evolutions of the Fourier modes are studied and plotted in Figure 4.9. The Fourier component $(1,0)$ is corresponding to the imposed two-dimensional TS wave disturbance at the inflow boundary with $\omega=0.0957$. The Fourier component $(1,2)$ is corresponding to the imposed harmonic three-dimensional TS wave disturbance at the inflow boundary with same temporal wave number as the two-dimensional TS wave.

From Figure 4.10, the flow transition is observed. The post transition skin friction is much closer to the turbulent flow. That shows the code has good grid convergence property. With grid resolution increase, we observe better results.

For this case, the inflow perturbation is relatively high, K-type transition is expected. From instantaneous flow field in Figure 4.11, a clear aligned pattern in the 
Table 4.6 Case 4 inflow parameters of finer grids

\begin{tabular}{cc}
\hline \hline & K-type \\
\hline$\alpha_{2 d}^{r}$ & 0.258 \\
$\alpha_{2 d}^{i}$ & $-6.72 \times 10^{-3}$ \\
$\omega_{2 d}$ & 0.0957 \\
$A_{2 d}$ & 0.02 \\
$\alpha_{3 d}^{r}$ & 0.258 \\
$\alpha_{3 d}^{i}$ & $-6.72 \times 10^{-3}$ \\
$\omega_{3 d}$ & 0.0957 \\
$A_{3 d}$ & 0.01 \\
$\beta$ & 0.2418 \\
$A_{r}$ & 0.01 \\
\hline \hline
\end{tabular}

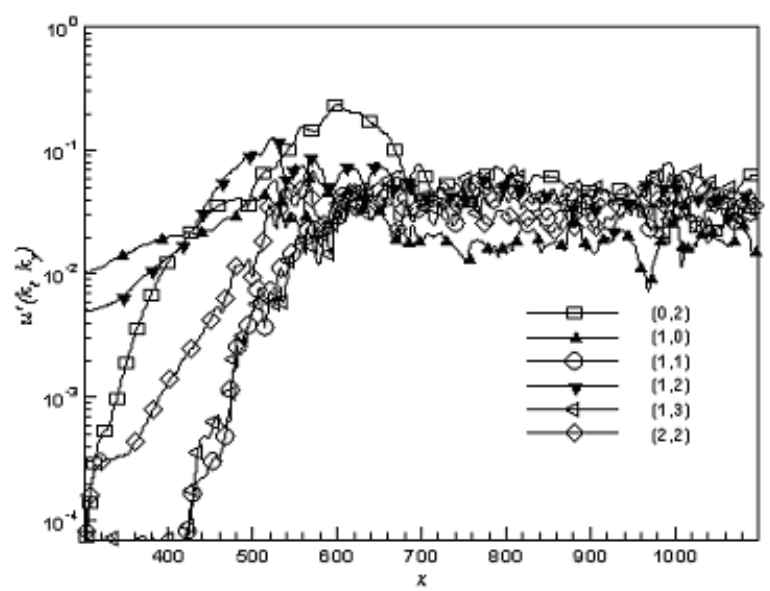

Figure 4.9 Case 4 spatial growth of streamwise perturbation

transition zone is observe. The $\Lambda$ shape vortical structures appear at $x=500 \delta_{\text {in }}$ and has an aligned pattern two wave length downstream. Afterward, the transition takes place and flow break down. This indicates a K-type transition. 


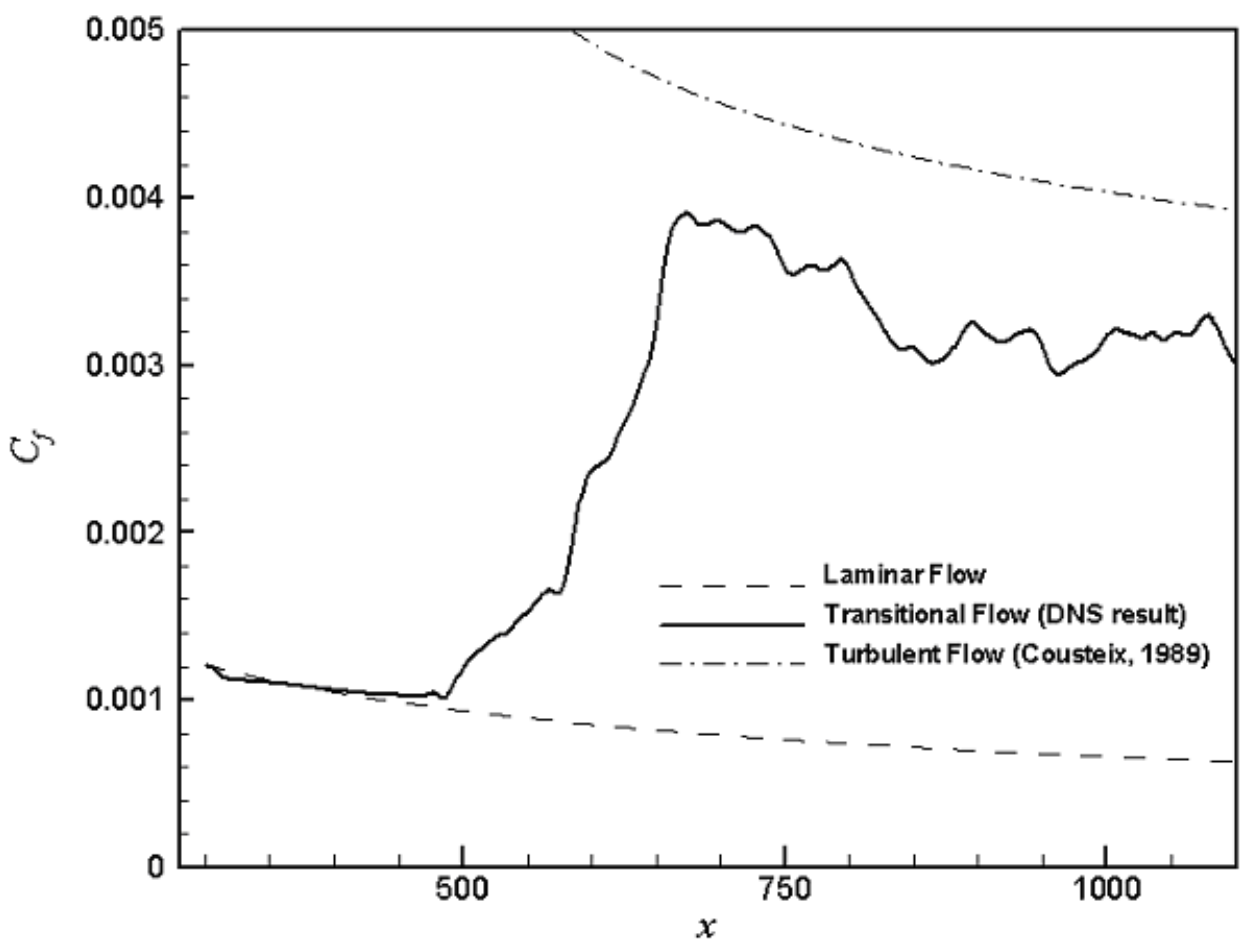

Figure 4.10 Case 4 skin friction comparison

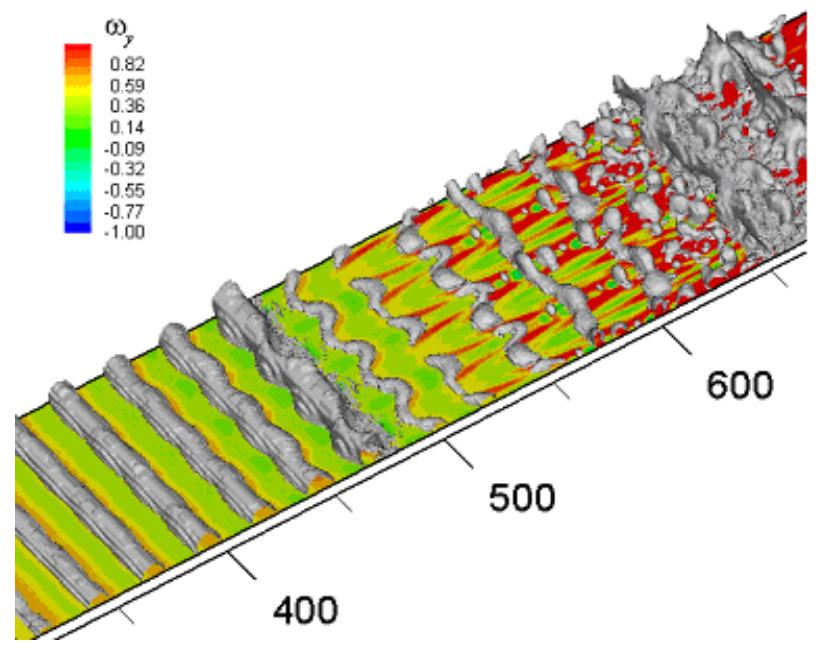

Figure 4.11 Case 4 instantaneous flow field 


\subsubsection{Finest grids $(1536 \times 128 \times 64)$}

\subsubsection{Case 5 - K-type transition in finest grid}

This case ran with the chosen parameters listed in table 4.7.

Table 4.7 Case 5 inflow parameters of finest grids

\begin{tabular}{cc}
\hline \hline & K-type \\
\hline$\alpha_{2 d}^{r}$ & 0.247 \\
$\alpha_{2 d}^{i}$ & $-6.611 \times 10^{-3}$ \\
$\omega_{2 d}$ & 0.0909 \\
$A_{2 d}$ & 0.02 \\
$\alpha_{3 d}^{r}$ & 0.247 \\
$\alpha_{3 d}^{i}$ & $-6.611 \times 10^{-3}$ \\
$\omega_{3 d}$ & 0.0909 \\
$A_{3 d}$ & 0.03 \\
$A_{r}$ & 0.01 \\
$\beta$ & 0.2 \\
\hline \hline
\end{tabular}

As previous cases, after the solution is well established in the whole domain, the spatial evolutions of the Fourier modes are studied and plotted in Figure 4.12. The Fourier component $(1,0)$ is corresponding to the imposed two-dimensional TS wave disturbance at the inflow boundary with $\omega=0.0909$. The Fourier component $(1,1)$ is corresponding to the imposed harmonic three-dimensional TS wave disturbance at the inflow boundary with same temporal wave number as the two-dimensional TS wave.

From Figure 4.13, the flow transition is observed. The post transition skin friction is closer to the turbulent flow. Again it confirms the code has good grid convergence property. With grid resolution increases, we observe better results. 


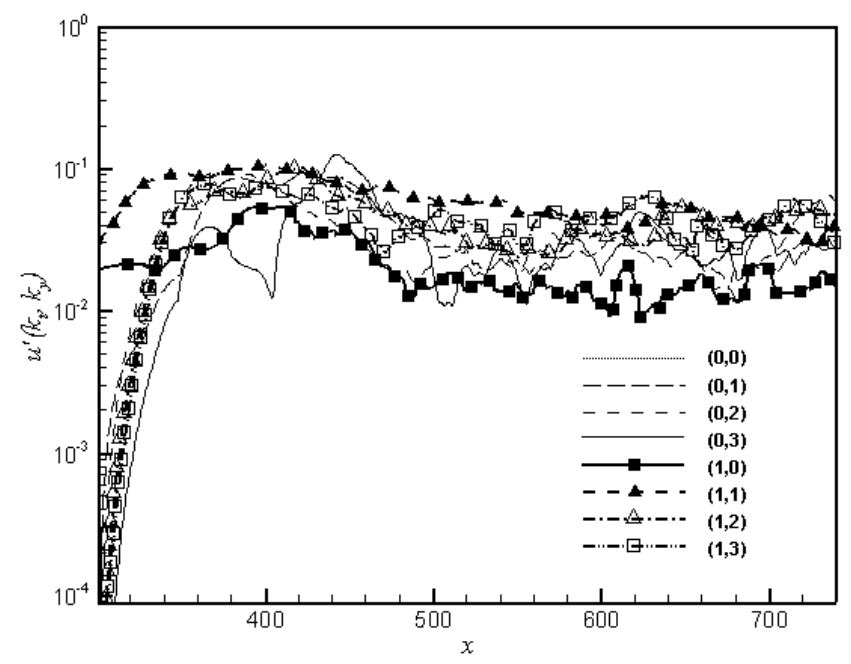

Figure 4.12 Case 5 spatial growth of streamwise perturbation

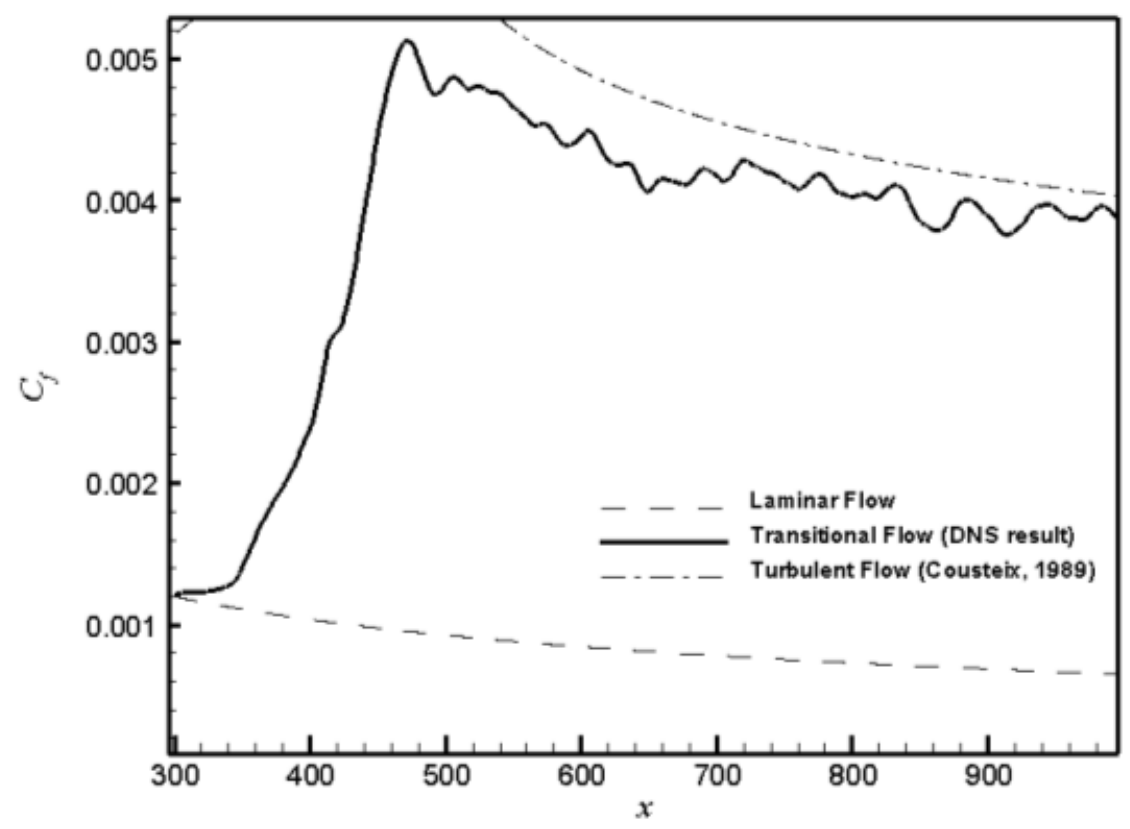

Figure 4.13 Case 5 skin friction comparison

For this case, the inflow perturbation is relatively high, K-type transition is expected. From instantaneous flow field in Figure 4.14, a clear aligned pattern in the transition zone is observed. The $\Lambda$ shape vortical structures appear at $x=350 \delta_{\text {in }}$ and 


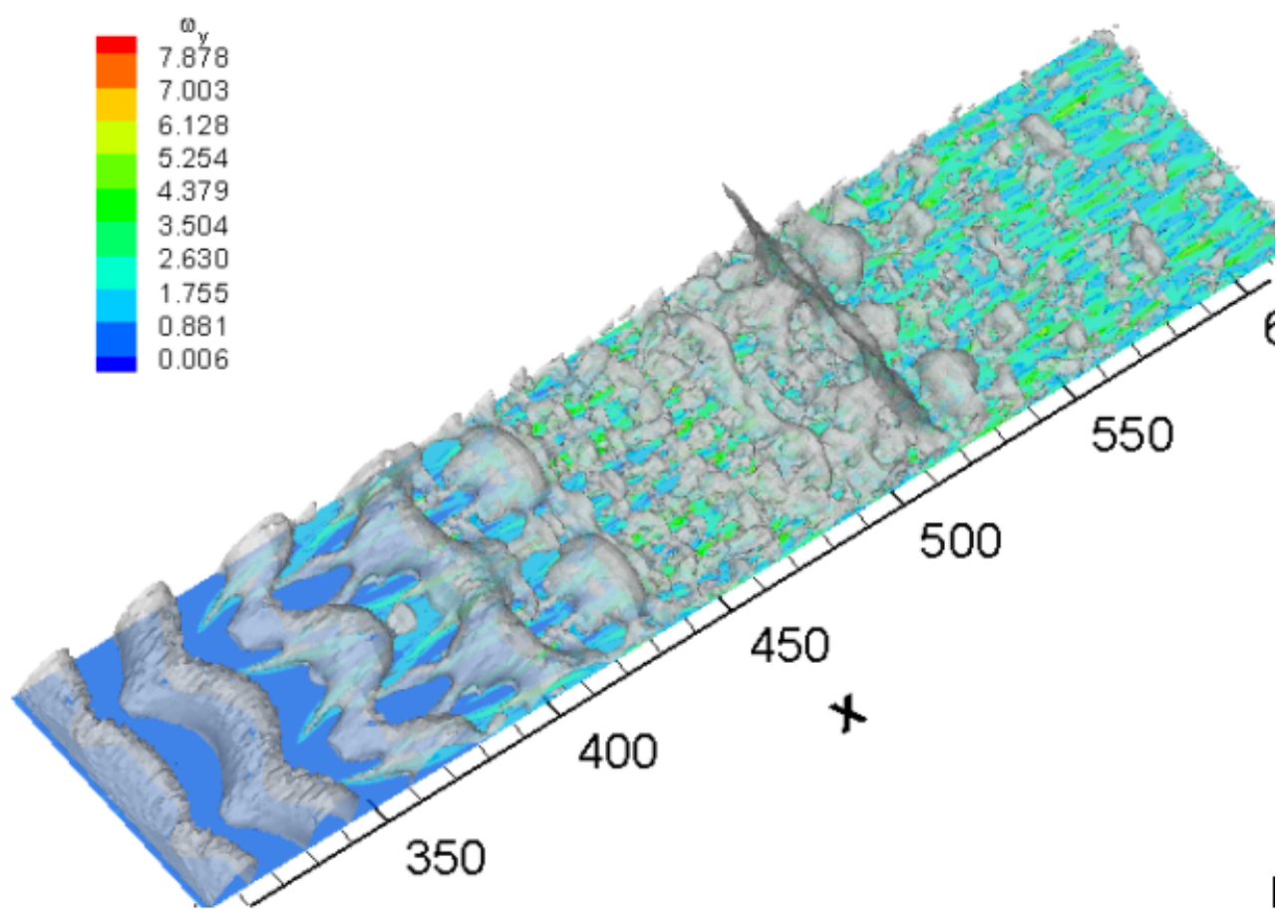

Figure 4.14 Case 5 instantaneous flow field

has an aligned pattern two wave length downstream. Afterward, the transition takes place and flow break down. This indicates a K-type transition.

\subsubsection{Case 6 - H-type transition in finest grid}

This case ran with the chosen parameters listed in table 4.8 .

As previous cases, after the solution is well established in the whole domain, the spatial evolutions of the Fourier modes are studied and plotted in Figure 4.15. The Fourier component $(1,0)$ is corresponding to the imposed two-dimensional TS wave disturbance at the inflow boundary with $\omega=0.0909$. The Fourier component $(1,1)$ is corresponding to the imposed harmonic three-dimensional TS wave disturbance at the inflow boundary with same temporal wave number as the two-dimensional TS wave. 
Table 4.8 Case 6 inflow parameters of finest grids

\begin{tabular}{cc}
\hline \hline & H-type \\
\hline$\alpha_{2 d}^{r}$ & 0.247 \\
$\alpha_{2 d}^{i}$ & $-6.611 \times 10^{-3}$ \\
$\omega_{2 d}$ & 0.0909 \\
$A_{2 d}$ & 0.02 \\
$\alpha_{3 d}^{r}$ & 0.247 \\
$\alpha_{3 d}^{i}$ & $-6.611 \times 10^{-3}$ \\
$\omega_{3 d}$ & 0.0909 \\
$A_{3 d}$ & 0.005 \\
$A_{r}$ & 0.005 \\
$\beta$ & 0.2 \\
\hline \hline
\end{tabular}

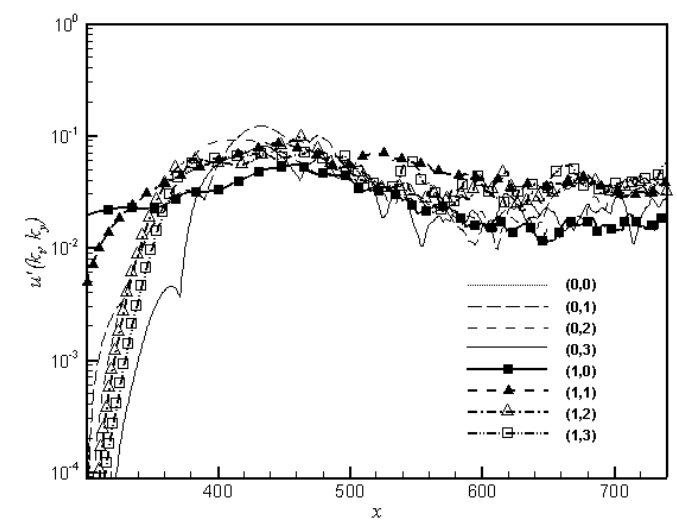

Figure 4.15 Case 6 spatial growth of streamwise perturbation

From Figure 4.16 transition is observed. As case 5, the post transition skin friction is much closer to the turbulent flow than the cases ran with insufficient grids resolution. For this case, the inflow perturbation is relatively low, H-type transition is expected. From instantaneous flow field in Figure 4.17, a clear staggered pattern in the transition zone is observe. The $\Lambda$ shape vortical structures appear at $x=360 \delta_{\text {in }}$ and has an aligned 


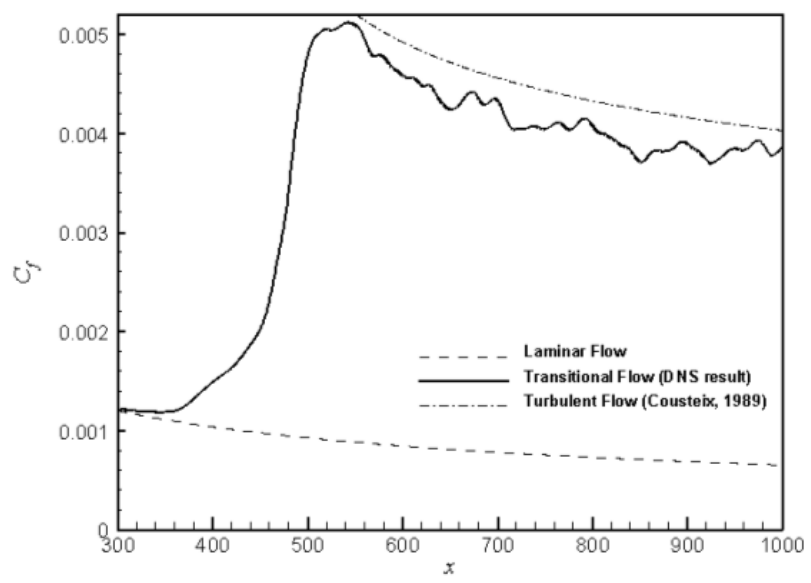

Figure 4.16 Case 6 skin friction comparison

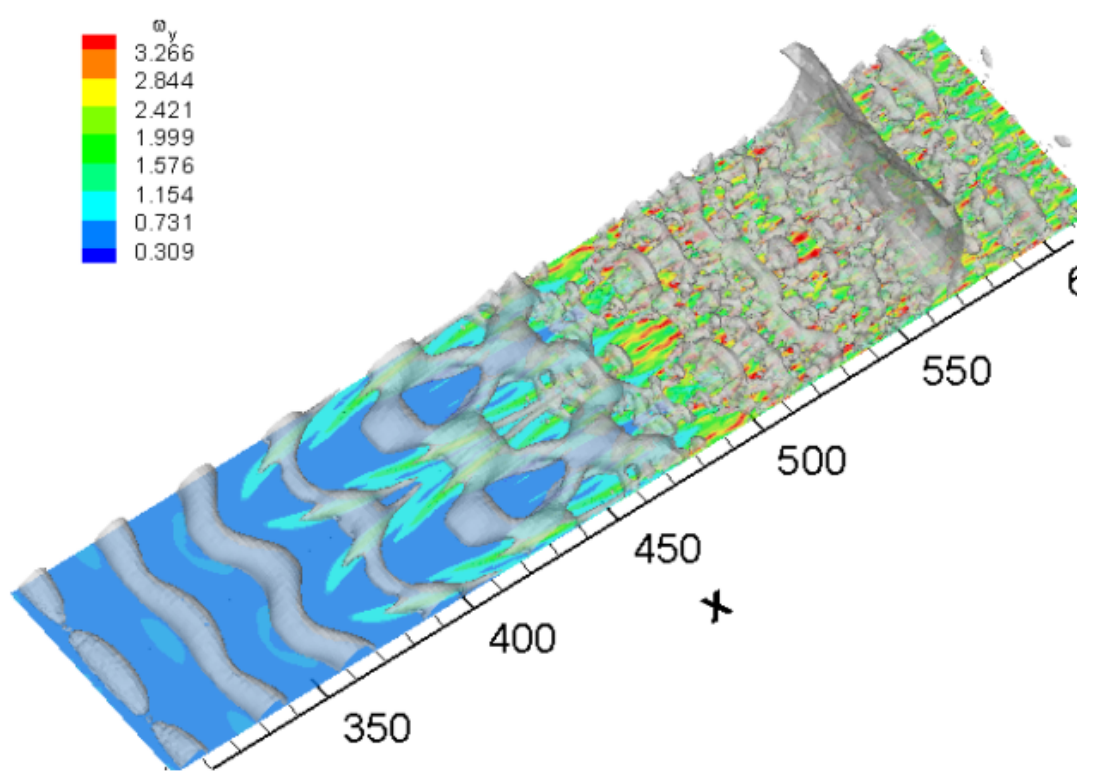

Figure 4.17 Case 6 instantaneous flow field

pattern two wave lengths downstream. Afterward, the staggered pattern takes place and flow break down. This indicates a H-type transition. 


\subsection{Discussion and Comparison}

\subsubsection{Skin friction comparison}

The skin friction coefficients calculated from the time- and spanwise-averaged velocity profiles for case 2, case 4, case 5 and case 6 are displayed in Figure 4.4,4.10,4.13 and 4.16 respectively. Computation results of Figure 4.4 and Figure 4.10 are coming from coarsest grid and finer grid, while Figure 4.13 and Figure 4.16 are from finest grids respectively. The spatial evolution of the skin friction coefficient of the laminar flow is plotted as dashed line in the figure. The empirical skin friction coefficient of a flat-plate turbulent boundary layer developed by Cousteix (1989) is given as:

$$
C_{f_{\text {tur }}}=0.0368 \operatorname{Re}_{\delta_{\text {in }}}^{-1 / 6}\left(\frac{x-x_{\text {in }}-l^{\prime}}{\delta_{\text {in }}}\right)^{-1 / 6}
$$

where $l^{\prime}=125 \delta_{i n}$. The empirical skin friction coefficient given by the above equation is also plotted as dash-doted line in all those figures as a comparison to the DNS result. It can be observed from Figure 4.13 that the $C_{f}$ curve starts with a laminar flow before $x=350 \delta_{\text {in }}$ in this simulation. A sudden growth in skin friction occurs afterward. That indicates the transition from laminar to turbulent flow happens. Around $x=500 \delta_{i n}$, the $C_{f}$ is very close to a turbulent value.

The post-transition level is comparable to that of fully developed turbulent flow. For both cases with finest grids, the post-transition friction values reach an almost same level. As for case 5, the transition point is at around $x=400 \delta_{i n}$. After that there is a rapid growth in skin friction. Comparing to the cases ran with coarse grids case 2 (Fig. 4.4) and case 4 (Fig. 4.10), the results of case 5 (Fig. 4.13) and case 6 (Fig. 4.13) are much better in comparison with the theoretical solution, with much less underestimate of the skin friction in the turbulent region. 


\subsubsection{Velocity profiles}

The time- and spanwise-averaged streamwise velocity profiles for various streamwise locations are displayed in Figure 4.18- Figure 4.21. The inflow velocity profile at $x=$ $311.40 \delta_{i n}$ is a typical laminar flow. At locations $x=725.22 \delta_{i n}$ and $x=1096.6 \delta_{i n}$, the velocity profiles are similar to that of fully developed turbulent flow. At location $x=406.9 \delta_{\text {in }}$, there is a small discrepancy between case 5 in Figure 4.20 and case 6 in Figure 4.21. For case 6, from Figure 4.21 we can see that it is just the beginning of the transition and the velocity profile is still very close to a laminar profile; but for case 5 , it is in the area of transition. In general, the velocity profiles and the skin frictions are well agreed.

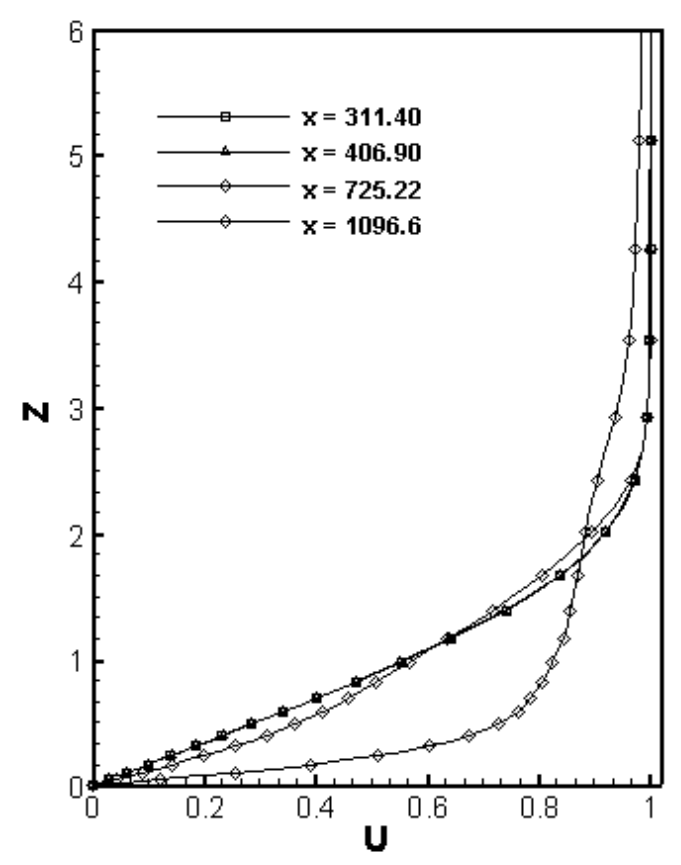

Figure 4.18 Velocity profiles for Case 2 


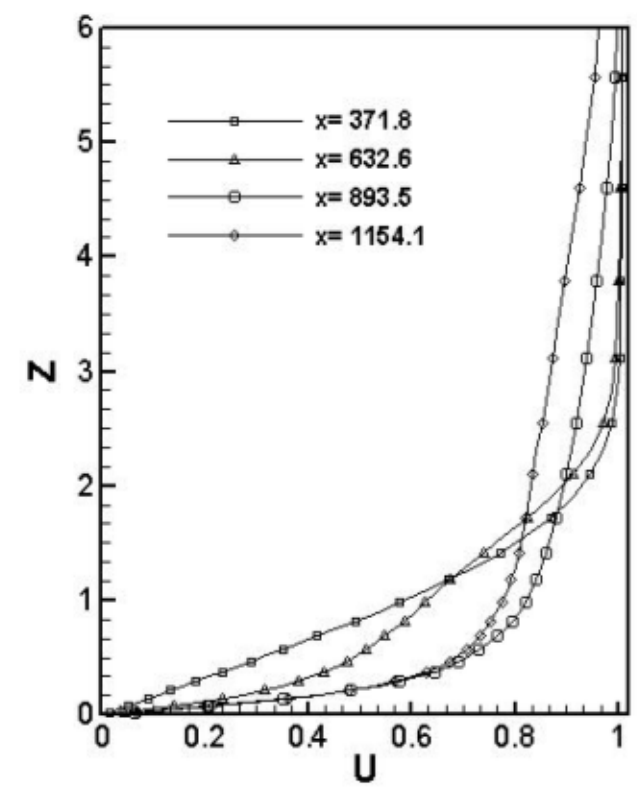

Figure 4.19 Velocity profiles for Case 4

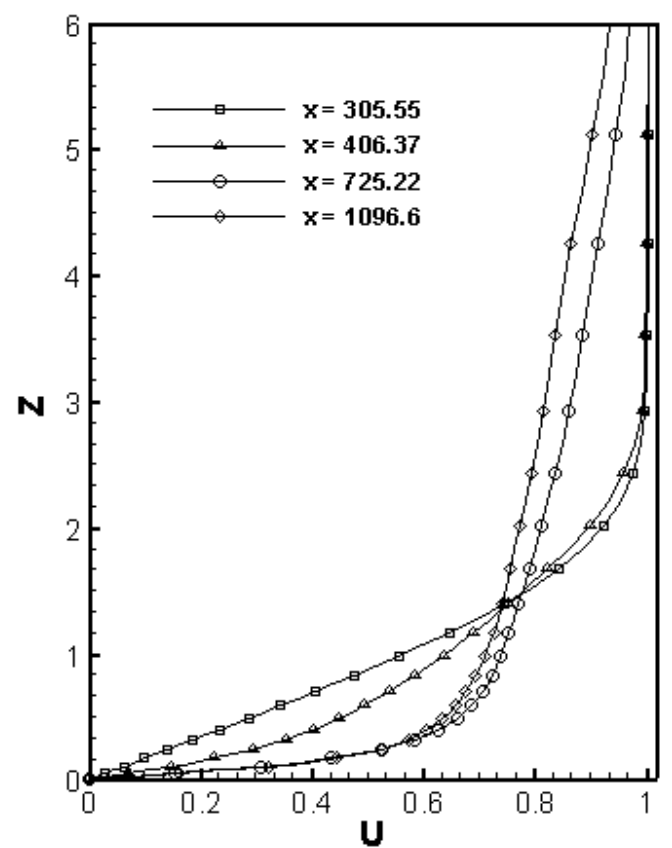

Figure 4.20 Velocity profiles for Case 5 


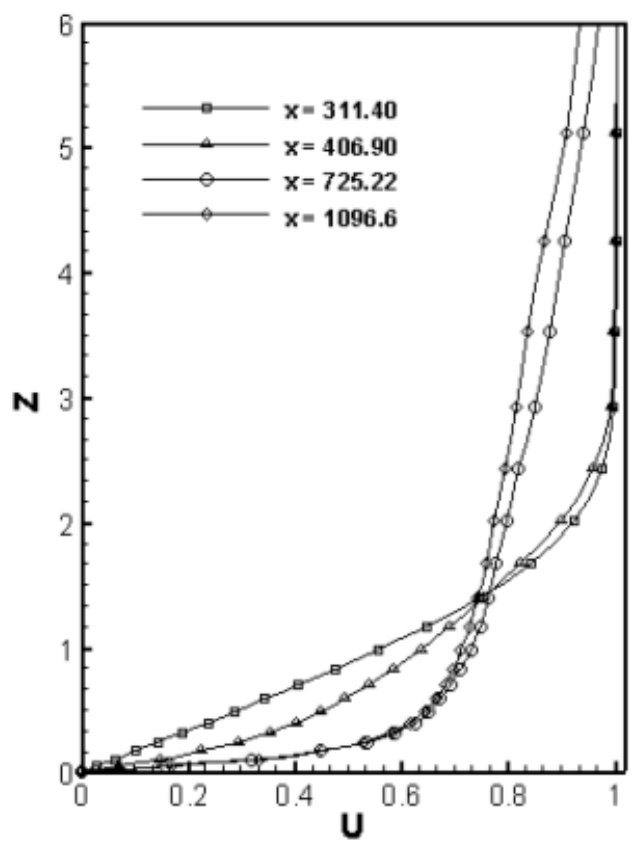

Figure 4.21 Velocity profiles for Case 6

In Figures 4.22 - 4.25 the velocity profiles at different streamwise locations are plotted in terms of logarithm scaled wall unit. The curves of the linear law and log law are also plotted in the same figure for comparison. Same as before Figure 4.22, 4.23, 4.24 and 4.25 are from different grid resolution levels. In all cases the velocity profiles are changed from laminar to turbulent profile when the streamwise location increases. Again, the results from Figure 4.24 and Figure 4.25 are in better agreement with the log law than the coarse grids and very consistent with previous results. For the results showing in Figure 4.22 and 4.23, it is reasonable when the coarse grids are used.

\subsubsection{Spectra and velocity statistics}

Figure 4.26 - Figure 4.29 show the spectra in $\mathrm{x}$ - and $\mathrm{y}$ - direction for both cases. The spectra are normalized by $\mathrm{z}$ at location of $R e_{x}=1.07 \times 10^{6}$ and $z^{+}=100,250$. Perry et al. approximately defined the turbulent region by $z^{+}>100$ and $z / \delta<0.15$. 


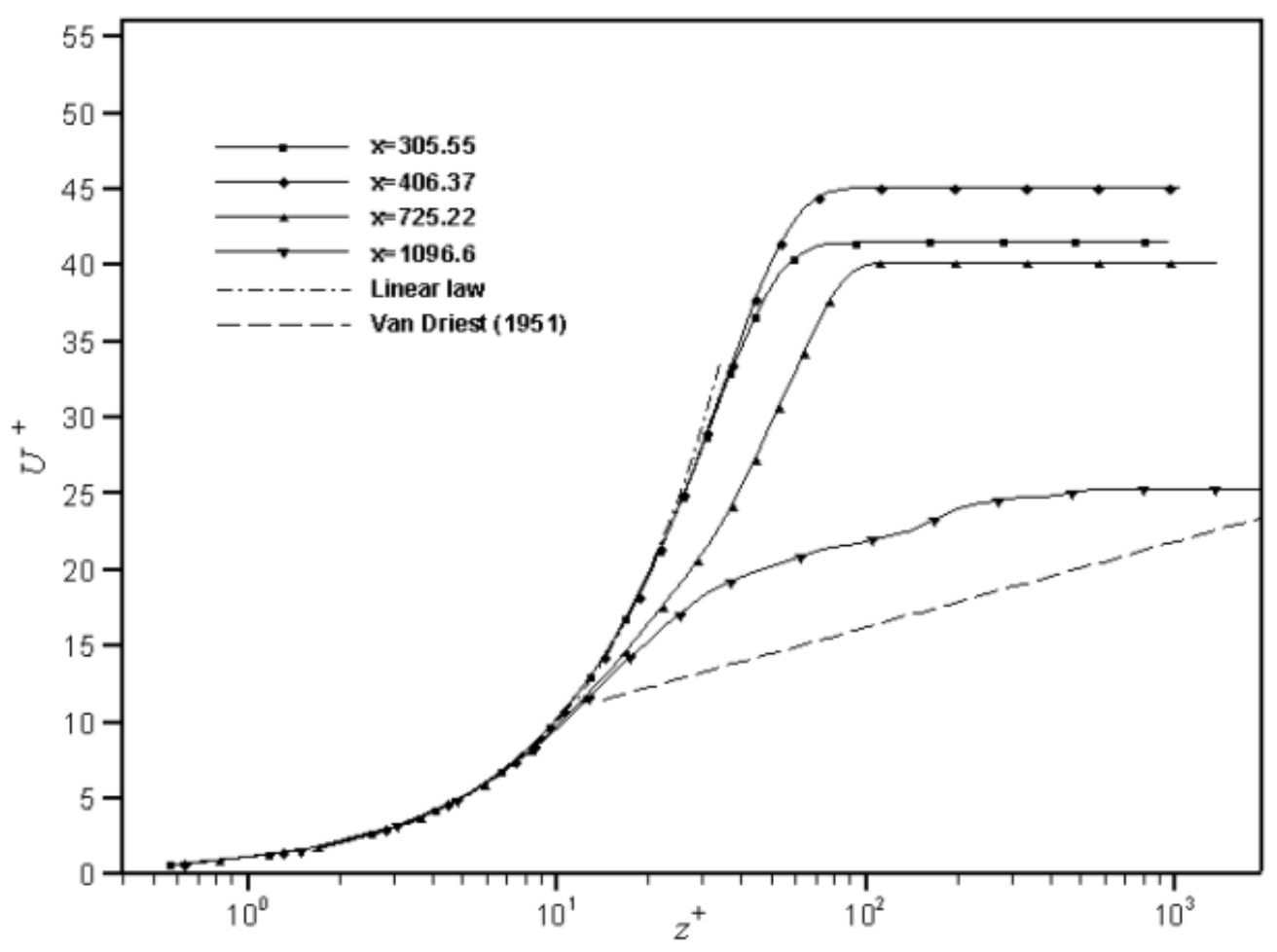

Figure 4.22 Log-linear plots for Case 2

In our case, for $R e_{x}=1.07 \times 10^{6}$ and $z / \delta \approx 0.15$ corresponds to $z^{+} \approx 350$, so the point $z^{+}=100,250$ should be in the turbulent region. A straight line with slope of $-\frac{5}{3}$ is also shown for comparison. The spectra tend to tangent to the $k^{-5 / 3}$ law. The large oscillations of the spectra can be attributed to the inadequate samples in time when the average is computed.

Figure 4.30 and 4.31 show Reynolds shear stress profile at various streamwise locations, which is normalized by square of wall shear velocity. For both cases, there are 10 streamwise locations from leading edge to trailing edge. As expected, close to the inlet where $R e_{x}=326.8 \times 10^{3}$ which is a laminar flow, the values of the Reynolds stress is much smaller then those in the turbulent region. For case 5 , the peak value increases with the increase of $\mathrm{x}$, around $R e_{x}=432.9 \times 10^{3}$ a big jump is observed in Figure 4.30, which 


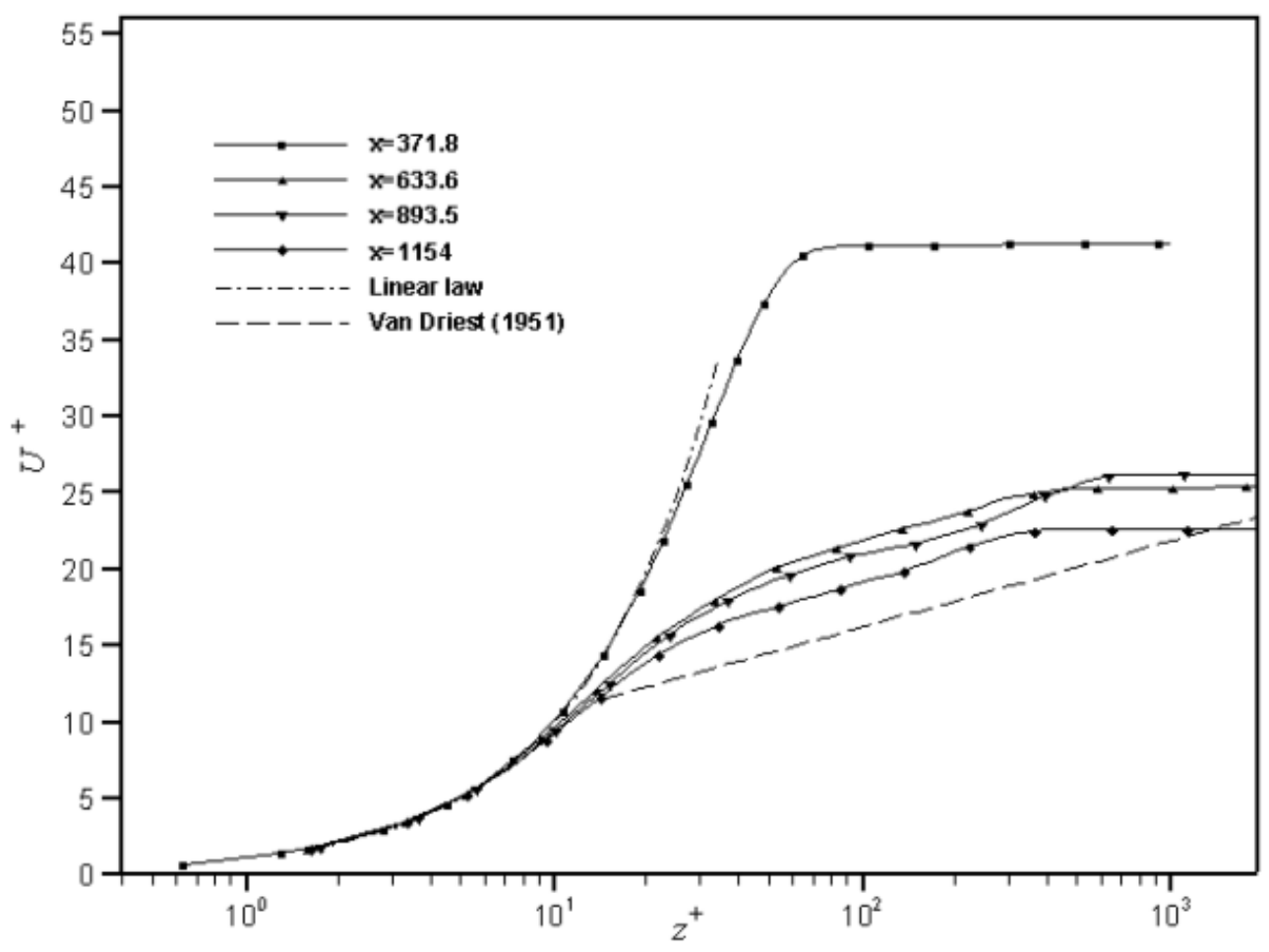

Figure 4.23 Log-linear plots for Case 4

indicates the flow is in transition. After the flow becomes turbulent, at $R e_{x}=485.9 \times 10^{3}$, the Reynolds stress comes close to the rest profiles. So for this case, we can consider after $R e_{x}=490 \times 10^{3}$, the flow become turbulence. While for case 6 , at $R e_{x}=485.9 \times 10^{3}$, there is a big jump in Reynolds stress and far away from the turbulence profile. When it comes to $R e_{x}=592 \times 10^{3}$, the Reynolds stress value becomes close to the turbulence value. That indicates the transition ends somewhere between $R e_{x}=485.9 \times 10^{3}$ and $R e_{x}=592 \times 10^{3}$. From Figure 4.31 it is clear that transition stops at $R e_{x}=540 \times 10^{3}$.

\subsubsection{Instantaneous flow field}

Figure 4.32 to 4.35 show the iso-surface of instantaneous low pressure and contours of the spanwise vorticity on the surface of the flat plate. Near the inflow boundary, small disturbance start to be visible. For case 5 in Figure 4.34, the disturbance grows 


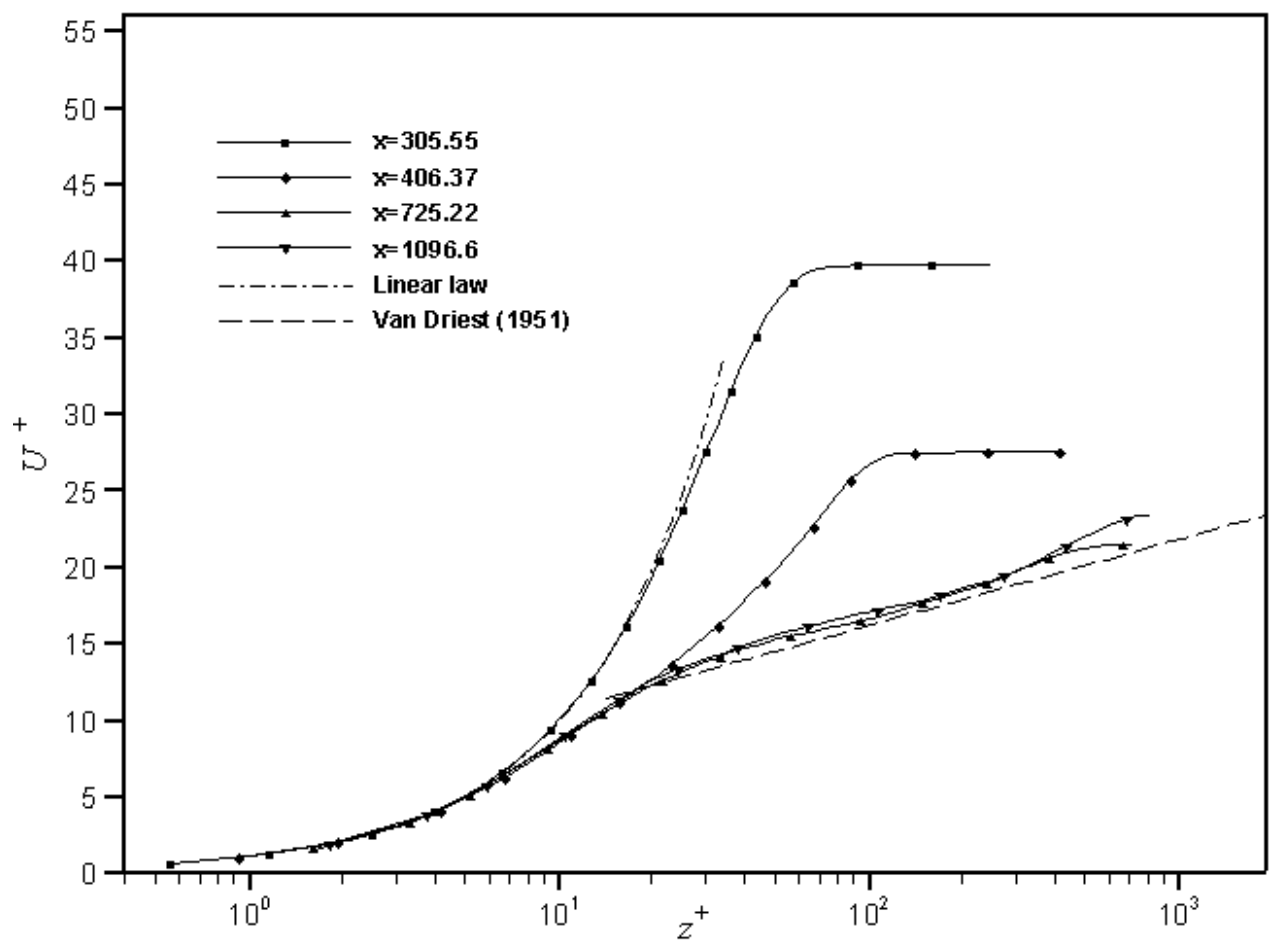

Figure 4.24 Log-linear plots for Case 5

rapidly between $x=300 \delta_{\text {in }}$ and $x=350 \delta_{\text {in }}$ where dramatic change is observed to the iso-surface of low pressure. $\Lambda$ shaped vortical structures appear near $x=330 \delta_{i n}$. At the same location, $\Lambda$ shaped strong shear layers can be seen from the contours of the spanwise vorticity on the surface of the flat plate. Before the location of $x=380 \delta_{i n}$, the aligned pattern is clear, that indicates a K-type transition. After the location of $x=400 \delta_{i n}$, the breakdown of $\Lambda$ shaped strong shear layers is visible. For case 6 in Figure 4.35, the transition happens further downstream. The disturbance grows rapidly between $x=320 \delta_{\text {in }}$ and $x=370 \delta_{\text {in }}$ where a dramatic change is observed to the iso-surface of low pressure. $\Lambda$ shaped vortical structures appear near $x=360 \delta_{i n}$. At the same location, $\Lambda$ shaped strong shear layers can be seen from the contours of the spanwise vorticity on the surface of the flat plate. Before the location of $x=460 \delta_{i n}$, the staggered pattern appears, 


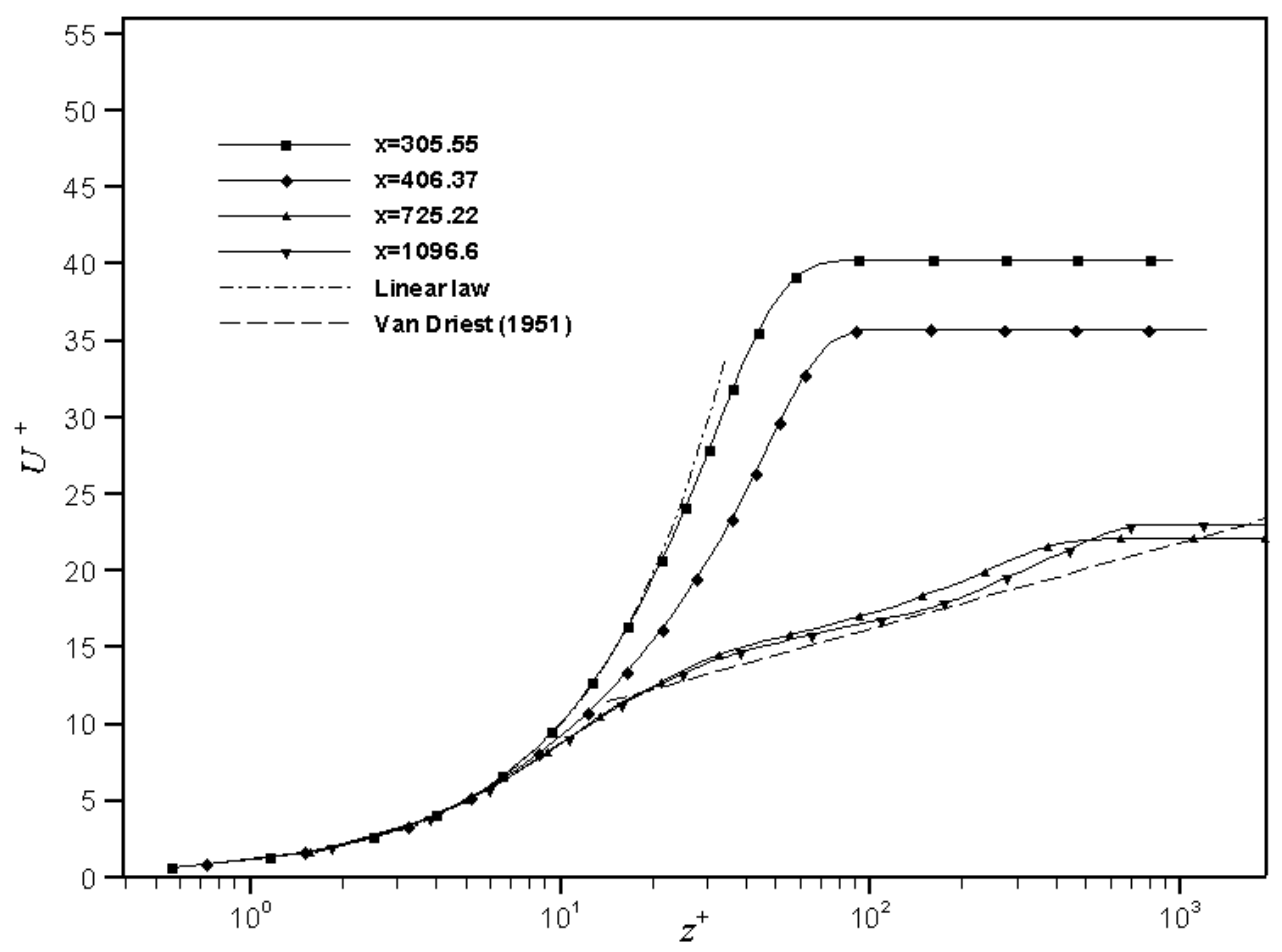

Figure 4.25 Log-linear plots for Case 6

that indicates a H-type transition. After the location of $x=480 \delta_{i n}$, the breakdown of shaped strong shear layers are visible.

For comparison, results on the coarse grids are also shown here. From Figure 4.4, $4.10,4.13$ and 4.16 we observed that the transition tends to happen in a larger interval for coarse grids. It could be caused by the grid viscosity. That also explains why we can see the aligned pattern (k-type Figure 4.32 and the staggered pattern (h-type Figure 4.33 much clearer. 


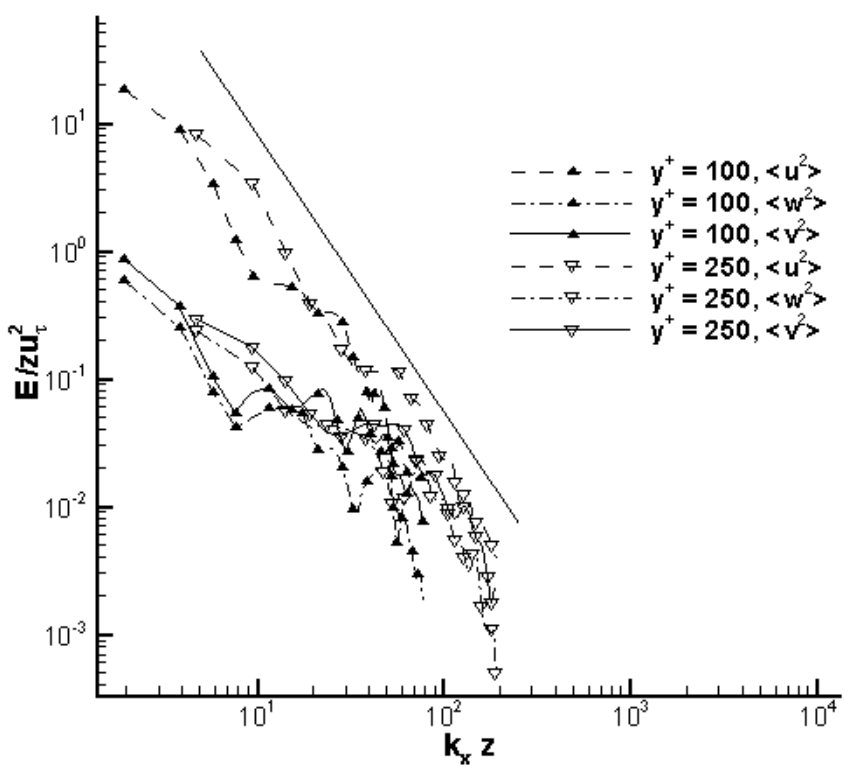

Figure 4.26 Spectra in x direction (Case 5)

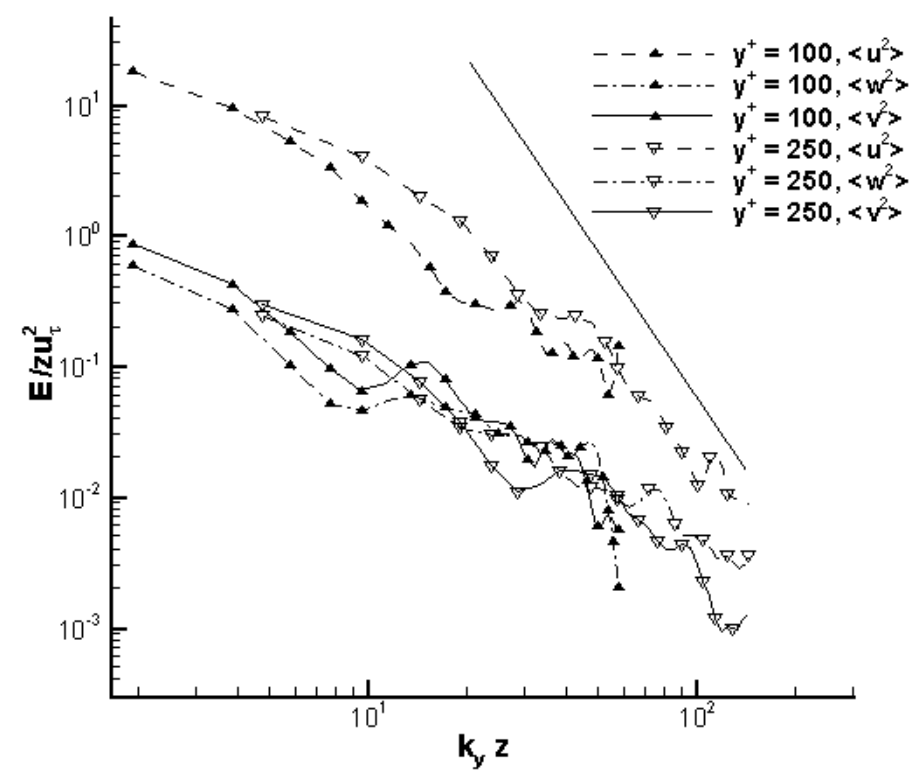

Figure 4.27 Spectra in $\mathrm{x}$ direction (Case 5) 


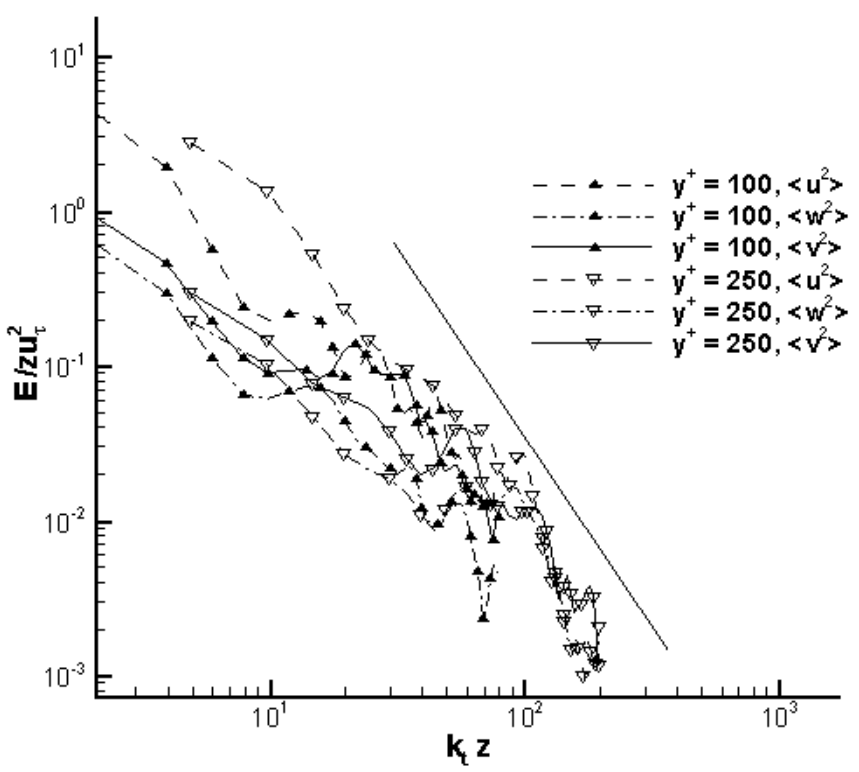

Figure 4.28 Spectra in x direction (Case 6)

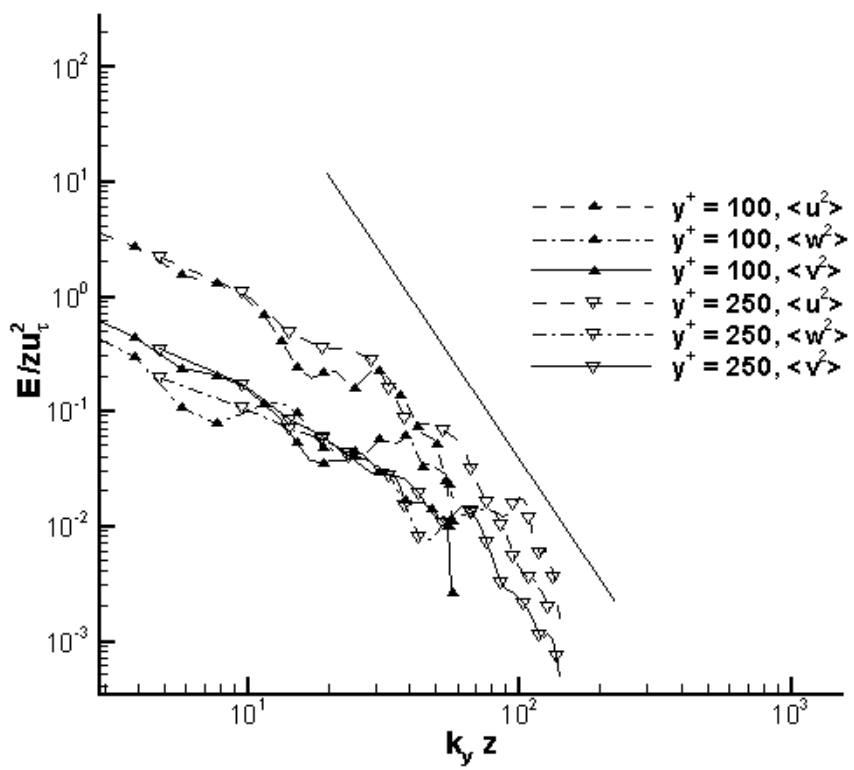

Figure 4.29 Spectra in $\mathrm{x}$ direction (Case 6) 


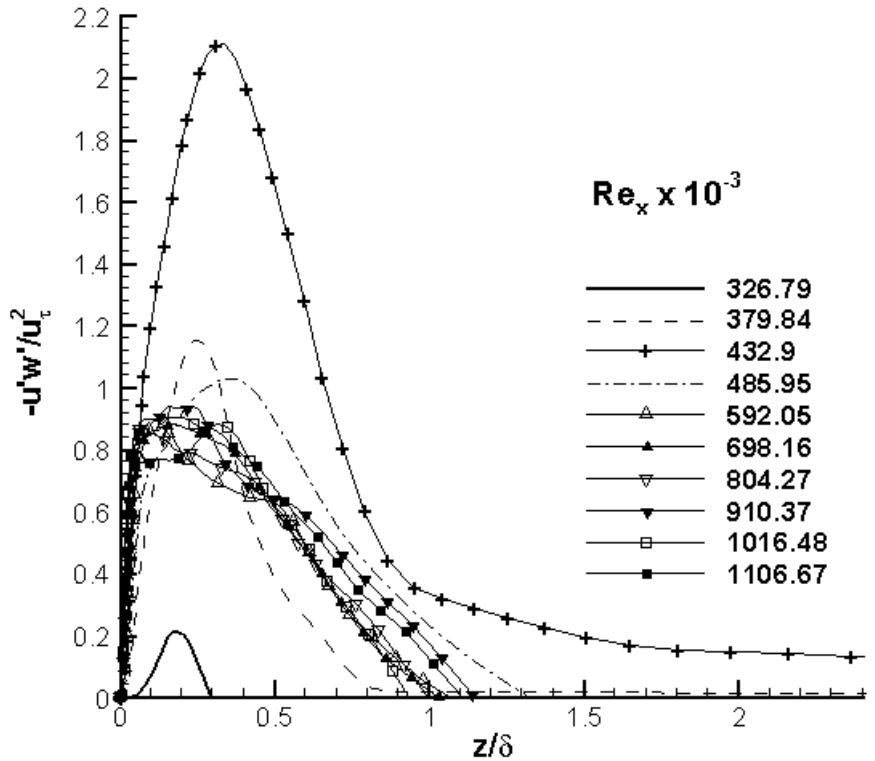

Figure 4.30 Reynolds stress (Case 5)

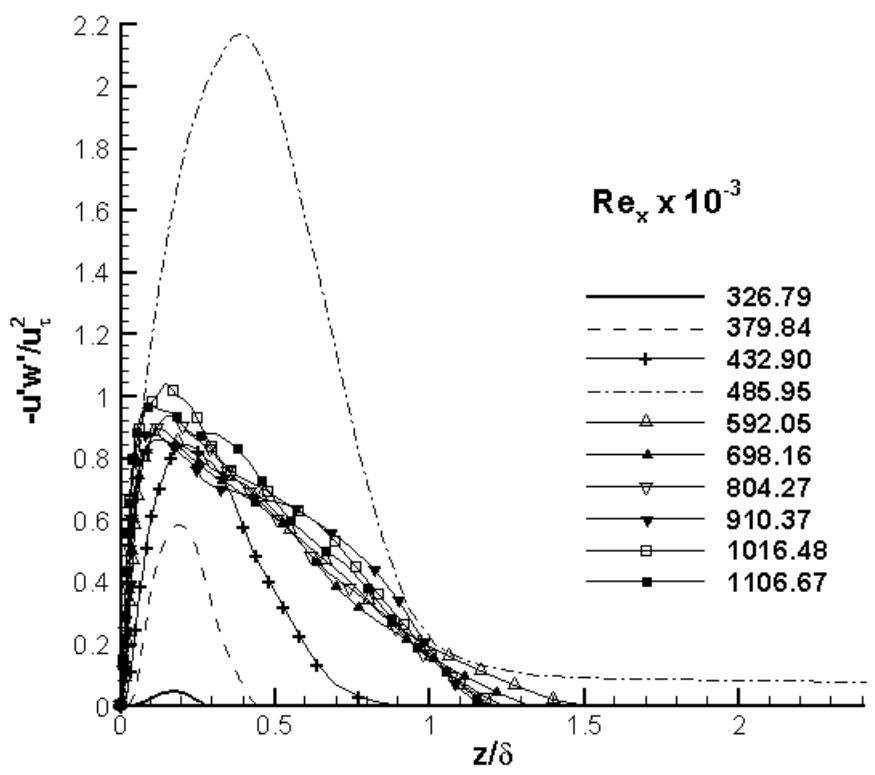

Figure 4.31 Reynolds stress (Case 6) 




Figure 4.32 Instantaneous flow field (Case 2)

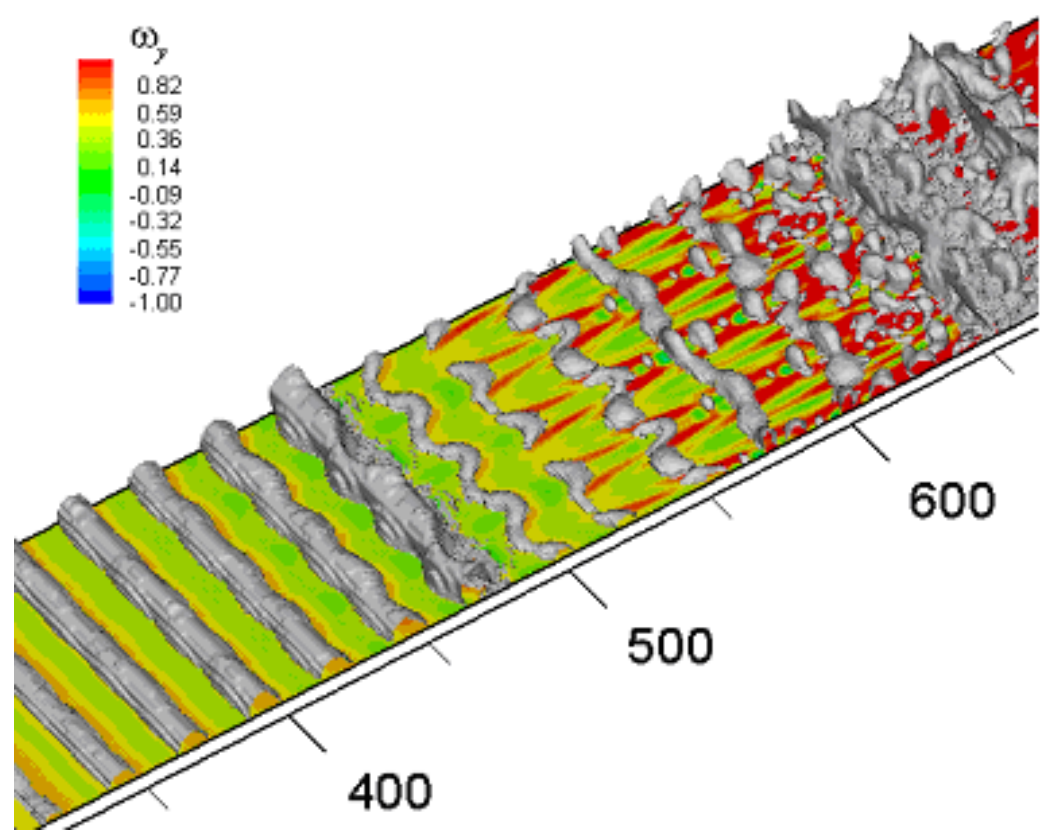

Figure 4.33 Instantaneous flow field (Case 4) 


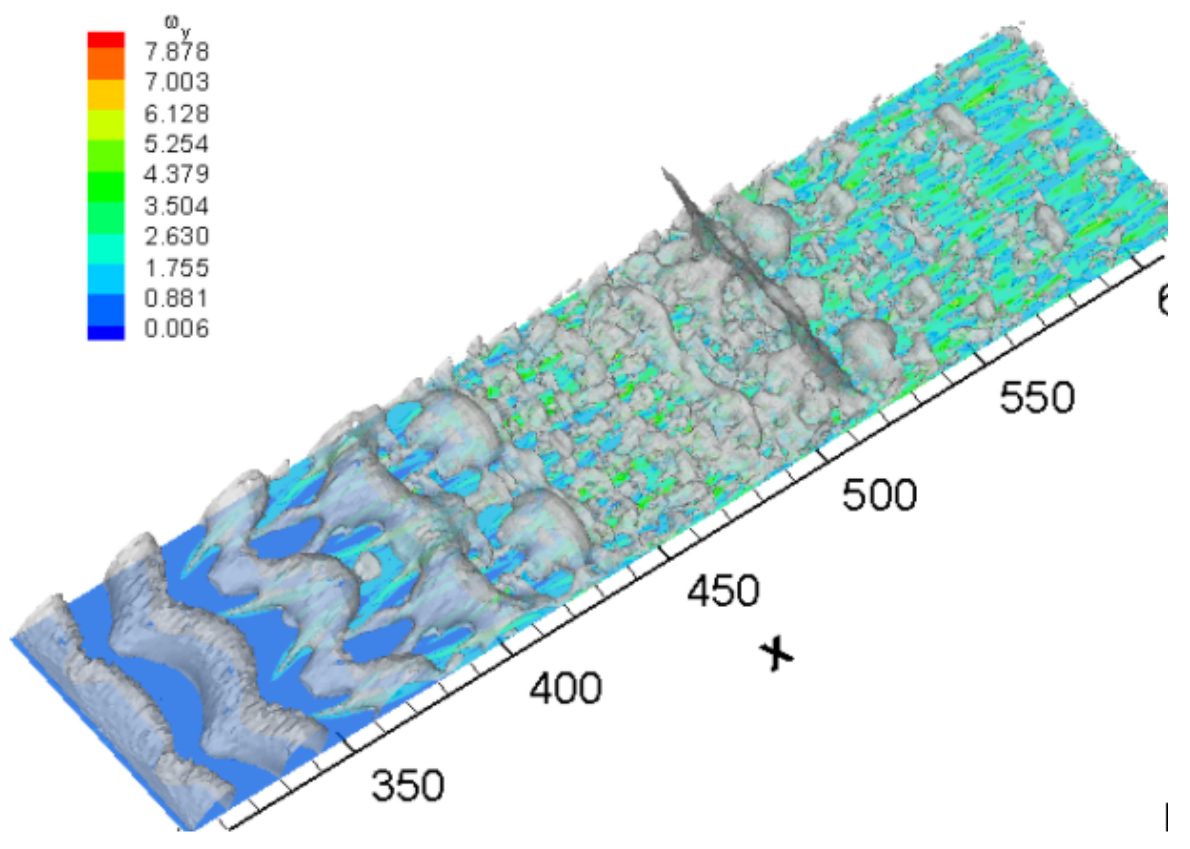

Figure 4.34 Instantaneous flow field (Case 5)

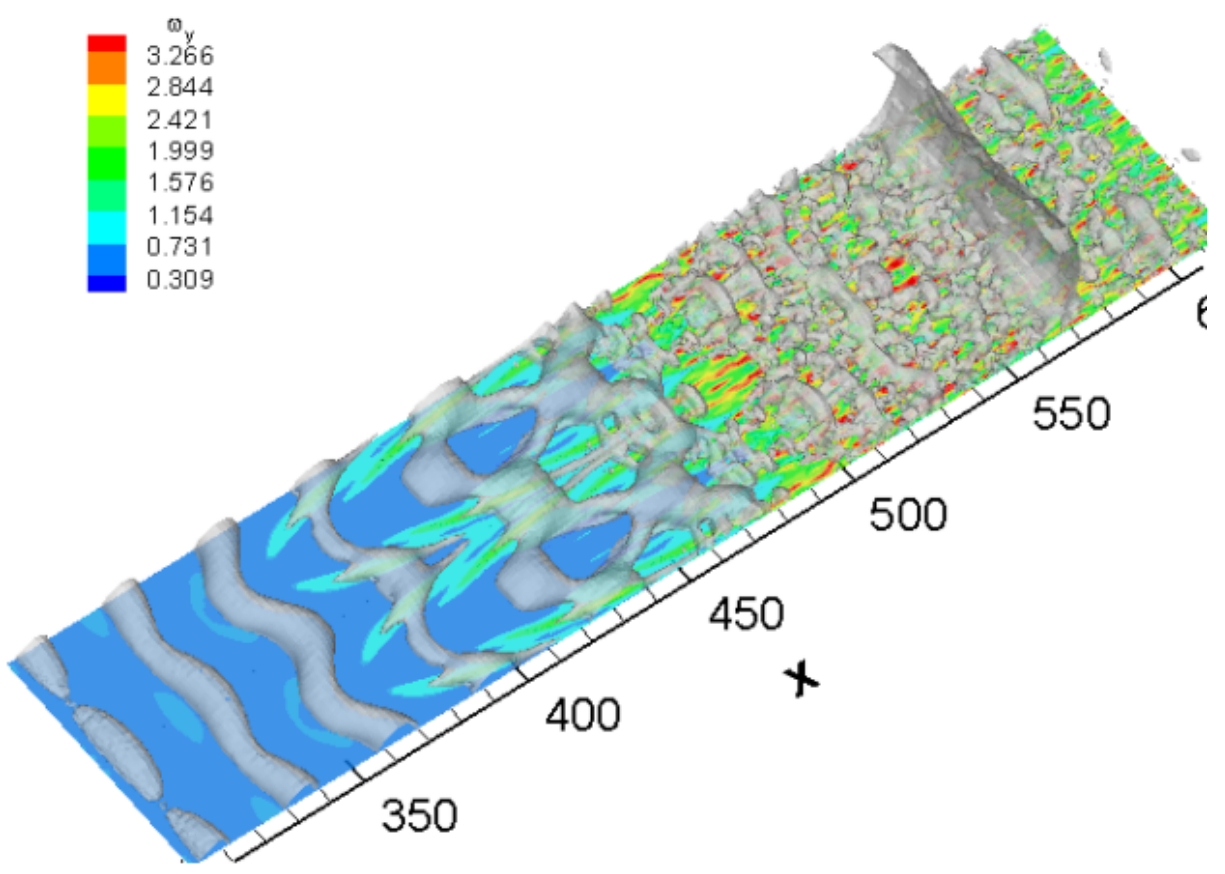

Figure 4.35 Instantaneous flow field (Case 6) 


\section{CHAPTER 5 CONCLUSIONS}

We have performed a direct numerical simulation of the complete transition to turbulence in a Mach number 0.5 boundary layer flow over a flat plate using the spatial approach. The results of six DNS cases are presented. These simulations show that the parallel computation is an efficient way for direct numerical simulation with the spatial approach. The numerical methods used in the simulation are successful. The nonreflecting boundary conditions work well at the far field and the outflow boundaries. The high-order compact finite difference scheme provides high accuracy and high resolution. By showing results from three different grids resolution, clear improvements are found when the grid resolution increases. Table 5.1 gives an overview of all the simulation cases ran. Base on the numerical simulation, we can conclude:

Table 5.1 Inflow disturbance parameters and transition type

\begin{tabular}{ccccccc}
\hline \hline & Case 1 & Case 2 & Case 3 & Case 4 & Case 5 & Case 6 \\
\hline$\omega_{2 d}$ & 0.12 & 0.0909 & 0.12 & 0.0957 & 0.0909 & 0.0909 \\
$A_{2 d}$ & 0.005 & 0.005 & 0.005 & 0.02 & 0.02 & 0.02 \\
$\omega_{3 d 1}$ & - & 0.0909 & 0.12 & 0.0957 & 0.0909 & 0.0909 \\
$A_{3 d 1}$ & - & 0.004 & 0.004 & 0.01 & 0.03 & 0.005 \\
$\omega_{3 d 2}$ & - & - & 0.06 & - & - & - \\
$A_{3 d 2}$ & - & - & 0.002 & - & - & - \\
$A_{r}$ & 0.008 & 0.002 & 0.002 & 0.01 & 0.01 & 0.005 \\
$\beta$ & - & 0.2 & 0.2 & 0.2418 & 0.2 & 0.2 \\
\hline Type of transition & No & H-type & H-type & K-type & K-type & H-type \\
\hline \hline
\end{tabular}


1. In order to observe transition, three-dimensional disturbance is needed, that agrees with the stability theory.

2.The TS wave linearly grows in certain range of Reynolds number, then it will damp out.

3. Three-dimensional disturbance can help keep the flow disturbance in growth and go to transition and turbulence for high Reynolds number.

$\mathrm{H}$ - or k-type transition is determined mainly by the $3 \mathrm{~d}$ disturbance amplitude. Case 5 and Case 6 are only different in the amplitude of three-dimensional perturbation which is imposed at the inflow boundary. For a larger three-dimensional perturbation in case 5, K-type transition observed. When a smaller three-dimensional perturbation is introduced in case 6, H-type transition is resulted. In both cases, transition to turbulence is observed, as shown from the averaged skin frictions plots and the log-linear plots. Same conclusion can be also reached by examining the power spectra and Reynolds shear stress. The result is comparable to the LES result of Ducros (1996). The transition process in Case 5 is is more rapid than that in case 6 , probably because that the amplitude of the perturbation at the inflow boundary is larger than that of Case 6 . 
APPENDIX A

\section{LAMINAR FLOW OVER A FLAT PLATE}


In this appendix, Blasius Equations is presented. Some numerical and experimental results related to laminar flow are also included

\section{A.1 Runge-Kutta Method for Blasius Equation}

Following is a FORTRAN subroutine of Runge-Kutta method for solving an arbitrary number of ordinary differential equations. It can be used to solve the Blasius equation (Eq. 2.15). The only changes needed are putting in different time step size $\mathrm{H}$ and the expression of the derivatives for different problem.

The subroutine is called by the FORTRAN with

$$
\text { CALL RUNGE(N,Y,F,X,H,M,K) }
$$

The arguments explanation follows:

$\mathrm{N}$ - Number of differential equations to be solved (set by the user for particular problem)

$\mathrm{Y} \quad$ - Array of $\mathrm{N}$ dependent variables (with initial value set by user)

$\mathrm{F} \quad$ - Array of $\mathrm{N}$ derivatives of variables $\mathrm{Y}$ (derivatives expression provided by user)

X - Independent variable (initialized by user)

H - Time step size (provided by user)

M - Index used in the subroutine which must be set to zero before the first CALL statement

$\mathrm{K}$ - Integer used in the program control

For example, a simple FORTRAN program to use this subroutine is as follows: 


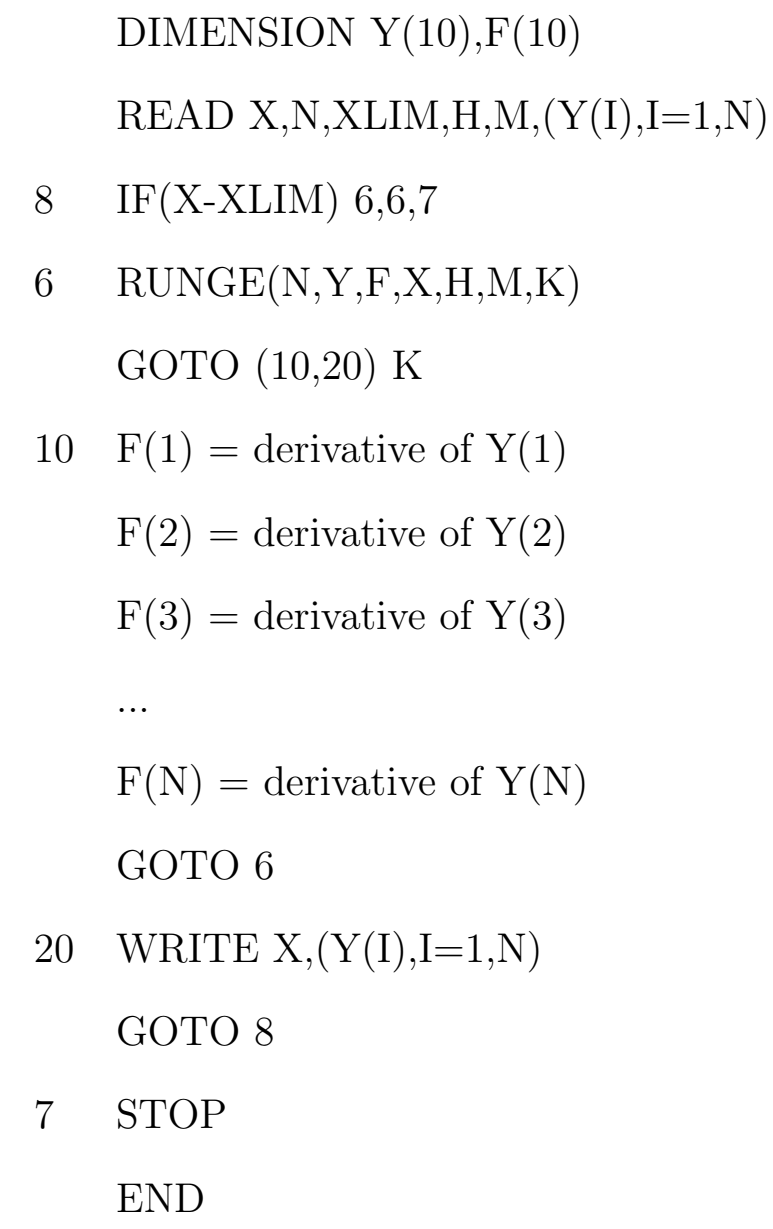

User needs to figure out the expression of $\mathrm{F}(\mathrm{I})$. There is no need to increase $\mathrm{x}$ in the main subroutine. One can change $\mathrm{H}$ at anytime. The array size of $\mathrm{Y}(\mathrm{I})$ and $\mathrm{F}(\mathrm{I})$ can be changed to any dimension users need.

The FORTRAN subroutine is described as follows 
SUBROUTINE RUNGE(N,Y,F,X,H,M,K)

DIMENSION Y(10), F(10), Q(10)

$$
\begin{aligned}
& \mathrm{M}=\mathrm{M}+1 \\
& \text { GO TO }(1,4,5,3,7) \mathrm{M} \\
& 1 \quad \mathrm{DO} 2 \mathrm{I}=1, \mathrm{~N} \\
& 2 \quad \mathrm{Q}(\mathrm{I})=0 . \\
& \mathrm{A}=.5 \\
& \text { GO TO } 9 \\
& 3 \quad \mathrm{~A}=1.707107 \\
& 4 \quad \mathrm{X}=\mathrm{X}+0.5 * \mathrm{H} \\
& 5 \quad \text { DO } 6 \mathrm{I}=1, \mathrm{~N} \\
& \mathrm{Y}(\mathrm{I})=\mathrm{Y}(\mathrm{I})+\mathrm{A}^{*}(\mathrm{~F}(\mathrm{I}) * \mathrm{H}-\mathrm{Q}(\mathrm{I})) \\
& 6 \mathrm{Q}(\mathrm{I})=2{ }^{*} \mathrm{~A}^{*} \mathrm{H}^{*} \mathrm{~F}(\mathrm{I})+\left(1 .-3 .{ }^{*} \mathrm{~A}\right) * \mathrm{Q}(\mathrm{I}) \\
& \mathrm{A}=.2928932 \\
& \text { GO TO } 9 \\
& 7 \quad \text { DO } 8 \mathrm{I}=1, \mathrm{~N} \\
& 8 \quad \mathrm{Y}(\mathrm{I})=\mathrm{Y}(\mathrm{I})+\mathrm{H}^{*} \mathrm{~F}(\mathrm{I}) / 6 \text {. }-\mathrm{Q}(\mathrm{I}) / 3 \text {. } \\
& \mathrm{M}=0 \\
& \mathrm{~K}=2 \\
& \text { GO TO } 10 \\
& 9 \quad \mathrm{~K}=1 \\
& 10 \text { RETURN } \\
& \text { END }
\end{aligned}
$$

Particularly, to use the above program to solve the Blasius equation

$$
2 f^{\prime \prime \prime}+f f^{\prime \prime}=0
$$


With the solution expression as follows

$$
\begin{gathered}
u=V_{\infty} f^{\prime}(\eta) \\
v=-\frac{1}{2} \sqrt{\frac{\nu V_{\infty}}{x}} f-\sqrt{\nu x V_{\infty}} \frac{\partial \eta}{\partial x} f^{\prime}
\end{gathered}
$$

where $\eta$ is the similarity variable

$$
\eta=y \sqrt{\frac{V_{\infty}}{\nu x}}
$$

Users need to solve for $\mathrm{f}$ which contain the only unknown $\eta$. Let $f^{\prime \prime}=Y 1, f^{\prime}=Y 2$ and $f=Y 3$, then the proper relations are

$$
\begin{array}{r}
F(1)=-Y(1) * Y(3) \\
F(2)=Y(1) \\
F(3)=Y(2)
\end{array}
$$

The remaining problem is to find the correct value $f^{\prime \prime}(0)=Y 1(\eta=0)$ which will make $f^{\prime}=Y 2$ approach to 1.0 as $\eta$ is going to infinity which is approximately 10.0. Practically we can treat $\eta=6.0$ as infinity. Then we can use computer to do a shooting. Starting by guessing the initial values of $f^{\prime \prime}(0)$, we can find the value $f^{\prime \prime}(0)$ which satisfies the condition $f^{\prime}(\infty)=Y 2(10.0)=1.0$. For example $f^{\prime \prime}(0)=0.332$ is an acceptable value. We can find the complete numerical solution of the Blasius equation from many other reference for example Schlichting(1979) and Anderson(1991) .

\section{A.2 Some Results of Laminar Flow from Blasius Solution}

From the Blasius solution, several important results regarding laminar flat-plate boundary layer can be derived.

Consider the local skin friction defined as $c_{f}=\frac{2 \tau_{w}}{\rho_{\infty} V_{\infty}^{2}}$, the wall shear stress is given by

$$
\tau_{w}=\mu \frac{\partial u}{\partial y}{ }_{y=0}
$$


Also we know that

$$
\frac{\partial u}{\partial y}=V_{\infty} \frac{\partial f^{\prime}}{\partial y}=V_{\infty} \sqrt{\frac{V_{\infty}}{\nu x}} \frac{f^{\prime}}{\eta}=V_{\infty} \sqrt{\frac{V_{\infty}}{\nu x}} f^{\prime \prime}
$$

Evaluate Eq.A.1 at the wall where $y=\eta=0$, we have the following

$$
\frac{\partial u}{\partial y}=V_{\infty=0} \sqrt{\frac{V_{\infty}}{\nu x}} f^{\prime \prime}(0)
$$

Substitute Eq.A.2 into the skin friction expression

$$
c_{f}=\frac{2 \tau_{w}}{\rho V_{\infty}^{2}}=\frac{2 \mu}{\rho V_{\infty}^{2}} V_{\infty} \sqrt{\frac{V_{\infty}}{\nu x}} f^{\prime \prime}(0)=2 \sqrt{\frac{\mu}{\rho_{\infty} V_{\infty} x}} f^{\prime \prime}(0)=\frac{2 f^{\prime \prime}(0)}{R e_{x}}
$$

Where $R e_{x}$ is the local Reynolds number. With $f^{\prime \prime}(0)=0.332$, we have

$$
c_{f}=\frac{0.664}{R e_{x}}
$$

This is a classic expression for the local skin friction coefficient for the incompressible laminar flow over a flat plate. Fig. A.2 shows the skin friction coefficient along a laminar flat plate.

Fig. A.3 shows the numerical solutions of second and third order DG method with the Blasius solution for a subsonic laminar flow. It can be observed that the skin friction coefficient approximation from boundary layer theory is very accurate. It can be shown that when the order of the numerical scheme is above third order, for such simple flow, they all come close to the Blasius approximation. For supersonic flow, in Zhong's paper (2003) a comparison between the Blasius approximation and numerical solution of up to $15^{\text {th }}$ order is given. It also concluded that the Blasius solution is very accurate.

By looking at the Fig. 2.1, it can be observed that at $\eta=5.0, u=0.99 V_{\infty}$, one may estimate the boundary-layer thickness, which is defined as the distance above the surface, where $u=0.99 V_{\infty}$, as:

$$
\eta=y \sqrt{\frac{V_{\infty}}{\nu x}}=\delta \sqrt{\frac{V_{\infty}}{\nu x}}=5.0
$$


That is

$$
\delta=\frac{5.0 x}{\sqrt{R e_{x}}}
$$

Displacement thickness is defined as

$$
\delta^{*}=\int_{0}^{y} 1\left(1-\frac{u}{V_{\infty}}\right) d y
$$

After variables transformation, it can be denoted in terms of f' and $\eta$ as

$$
\delta^{*}=\sqrt{\frac{\nu x}{V_{\infty}}} \int_{0}^{\eta_{1}}\left(1-f^{\prime}(\eta)\right) d \eta=\sqrt{\frac{\nu x}{V_{\infty}}}\left[\eta_{1}-f\left(\eta_{1}\right)\right]
$$

From the numerical solution, for all value of $\eta>5.0, \eta_{1}-f\left(\eta_{1}\right)=1.72$, therefore,

$$
\delta^{*}=\frac{1.72 x}{\sqrt{R e_{x}}}
$$

Follow similar procedure, the momentum thickness is estimated as

$$
\theta=\frac{0.664 x}{\sqrt{R e_{x}}}
$$




\begin{tabular}{llll}
\hline $\boldsymbol{\eta}$ & $\boldsymbol{f}(\boldsymbol{\eta})$ & $\boldsymbol{f}^{\prime}(\boldsymbol{\eta})$ & $\boldsymbol{f}^{\prime \prime}(\boldsymbol{\eta})$ \\
\hline 0.0 & 0.0 & 0.0 & 0.46960 \\
0.1 & 0.00235 & 0.04696 & 0.46956 \\
0.2 & 0.00939 & 0.09391 & 0.46931 \\
0.3 & 0.02113 & 0.14081 & 0.46861 \\
0.4 & 0.03755 & 0.18761 & 0.46725 \\
0.5 & 0.05864 & 0.23423 & 0.46503 \\
0.6 & 0.08439 & 0.28058 & 0.46173 \\
0.7 & 0.11474 & 0.32653 & 0.45718 \\
0.8 & 0.14967 & 0.37196 & 0.45119 \\
0.9 & 0.18911 & 0.41672 & 0.44363 \\
1.0 & 0.23299 & 0.46063 & 0.43438 \\
1.1 & 0.28121 & 0.50354 & 0.42337 \\
1.2 & 0.33366 & 0.54525 & 0.41057 \\
1.3 & 0.39021 & 0.58559 & 0.39598 \\
1.4 & 0.45072 & 0.62439 & 0.37969 \\
1.5 & 0.51503 & 0.66147 & 0.36180 \\
1.6 & 0.58296 & 0.69670 & 0.34249 \\
1.7 & 0.65430 & 0.72993 & 0.32195 \\
1.8 & 0.72887 & 0.76106 & 0.30045 \\
1.9 & 0.80644 & 0.79000 & 0.27825 \\
2.0 & 0.88680 & 0.81669 & 0.25567 \\
2.2 & 1.05495 & 0.86330 & 0.21058 \\
2.4 & 1.23153 & 0.90107 & 0.16756 \\
2.6 & 1.41482 & 0.93060 & 0.12861 \\
2.8 & 1.60328 & 0.95288 & 0.09511 \\
3.0 & 1.79557 & 0.96905 & 0.06771 \\
3.2 & 1.99058 & 0.98037 & 0.04637 \\
3.4 & 2.18747 & 0.98797 & 0.03054 \\
3.6 & 2.38559 & 0.99289 & 0.01933 \\
3.8 & 2.58450 & 0.99594 & 0.01176 \\
4.0 & 2.78388 & 0.99777 & 0.00687 \\
4.2 & 2.98355 & 0.99882 & 0.00386 \\
4.4 & 3.18338 & 0.99940 & 0.00208 \\
4.6 & 3.38329 & 0.99970 & 0.00108 \\
4.8 & 3.58325 & 0.99986 & 0.00054 \\
5.0 & 3.78323 & 0.99994 & 0.00026 \\
5.2 & 3.98322 & 0.999971 & 0.000119 \\
5.4 & 4.18322 & 0.999988 & 0.000052 \\
5.6 & 4.38322 & 0.999995 & 0.000022 \\
5.8 & 4.58322 & 0.999998 & 0.000009 \\
6.0 & 4.78322 & 0.999999 & 0.000003 \\
\hline & & & \\
\hline
\end{tabular}

Figure A.1 Blasius solution 


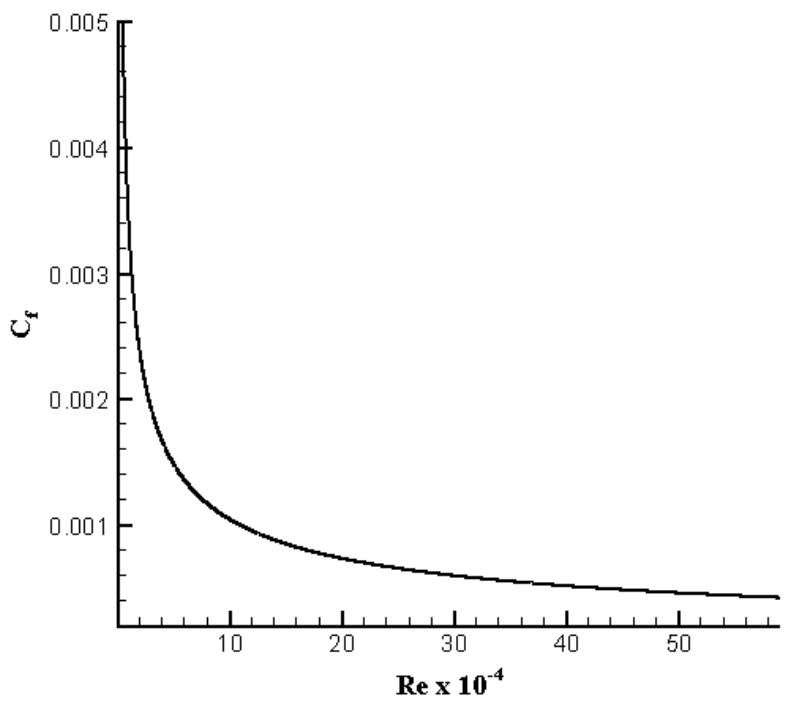

Figure A.2 Skin friction from Blasius solution

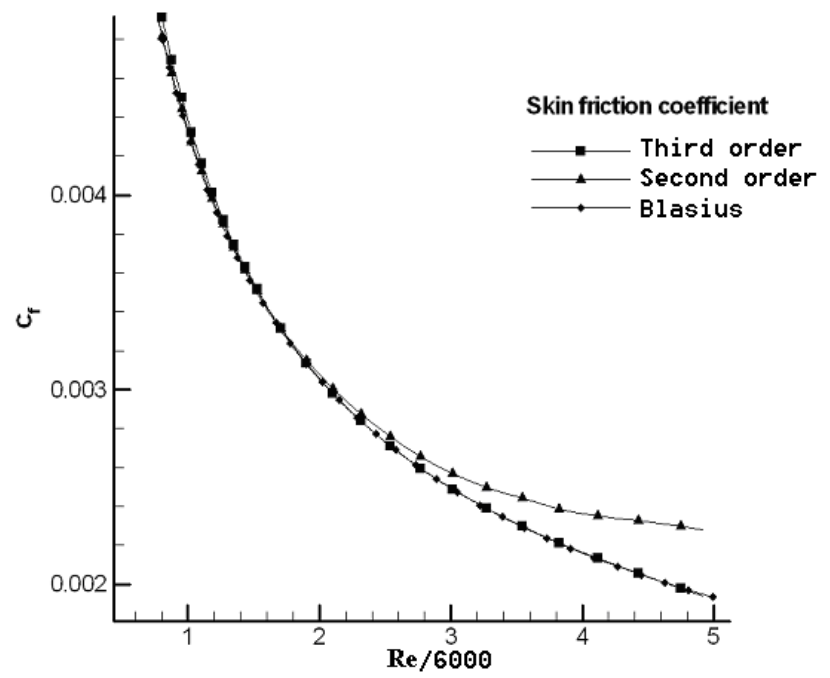

Figure A.3 Comparison between DG scheme and Blasius approximation 


\section{REFERENCES}

[1] C. W. Shu and S. Osher, "Efficient implementation of essentially non-oscillatory shock capturing scheme," Journal of Computational Physics, vol. 77, 1988.

[2] S. Lele, "Compact finite difference schemes with spectral-like resolution," J. Comp. Phys., vol. 16, 1992.

[3] L. Jiang, H. Shan, and C. Liu, "Non-reflecting boundary conditions for dns in curvilinear coordinates," in Recent Advances in DNS and LES-Proceedings of the Second AFOSR International Conference on DNS/LES, Rutgers-The State University of New Jersey, New Brunswick, June 1999.

[4] _ _ "Parallel direct numerical simulation of flow transition in boundary layers," UTA, Arlington, TX, NASA Report, Dec. 2001.

[5] H. Shan, L. Jiang, and C. Liu, "Direct numerical simulation of flow separation around a naca 0012 airfoil," Journal of Computer and Fluids, 2005.

[6] P. Klebanoff, K. Tidstrom, and L. Sargent, "The three dimensional nature of boundary layer instability," J. Fluid Mech., vol. 12, 1962.

[7] T. Herbert, "Three-dimensional phenomena in the transitional flat-plate boundary layer," in AIAA paper, 1985-0489.

[8] C. F. Knapp and P. J. Roache, "A combined visual and hot-wire anemometer investigation of boundary-layer transition," AIAA Journal, vol. 6, 1968.

[9] K. Y. S. and L. V. Ya., "The resonant interaction of disturbances at laminarturbulent transition in a boundary layer." J. Fluid Mech, vol. 138, 1962. 
[10] R. D. Joslin, C. L. Streett, and C.-L. Chang, "Spatial direct numerical simulation of boundarylayer transition mechanisms: Validation of pse theory," Theoret. Comput. Fluid Dyn, vol. 4, 1993.

[11] L. Jiang, H. Shan, and C. Liu, "Direct numerical simulation of boundary-layer receptivity for subsonic flow around airfoil," in Recent Advances in DNS and LESProceedings of the Second AFOSR International Conference on DNS/LES, RutgersThe State University of New Jersey, New Brunswick, June 1999.

[12] D. Benney and C. C. Lin, "On the secondary motion induced by oscillations in a shear flow," Phys. Fluids, vol. 3, 1960.

[13] F. Ducros, P. Comte, and M. Lesieur, "Large-eddy simulation of transition to turbulence in a boundary layer developing spatially over a flat plate," J. Fluid Mech, vol. 1, 1996.

[14] M. El-hady and T. A. Zang, "Large-eddy simulation of nonlinear evolution and breakdown to turbulence in high-speed boundary layers," Theo. Comp. Fluid Dynamics, vol. 7, 1995.

[15] G. Erlebacher and M. Y. Hussaini, "Numerical experiments in supersonic boundarylayer stability," Theo. Comp. Fluid Dynamics, 1990.

[16] P. A. Elofsson and P. H. Alfredsson, "An experimental study of oblique transition in plane poiseuille flow," J. Fluid Mech, vol. 358, 1998.

[17] K. Y. S. and L. V. Ya., "Nonlinear development of a wave in a boundary layer," Fluid Dynamics, vol. 12, 1978.

[18] S. Berlin, M. Wiegel, and D. S. Henningson, "Numerical and experimental investigations of oblique boundary layer transition," J. Fluid Mech, vol. 393, 1999.

[19] P. A. Elofsson and P. H. Alfredsson, "An experimental study of oblique transition in a blasius boundary layer flow," Eur. J. Mech. B/Fluids, vol. 19, 2000. 
[20] K. S. Breuer, J. Cohen, and J. H. Haritonidis, "The late stages of transition induced by a low-amplitude wavepacket in a laminar boundary layer," J. Fluid Mech, vol. $340,1997$.

[21] E. R. Van Driest, "Turbulent boundary layer in compressible fluids," J. Aero Sci., vol. 18, 1951.

[22] M. Visbal and D. Gaitonde, "High-order accurate methods for unsteady vortical flows on curvilinear meshes," in AIAA paper, Reno, Nevada, Jan 1998.

[23] D. Gaitonde and M. Visbal, "Further development of a navier-stokes solution procedure based on higher-order formulas," in AIAA Paper 99-0557, Reno, Nevada, Jan 1999.

[24] T. Zang and M. Hussanini, "Multiple paths to subharmonic laminar breakdown in a boundary layer," Phys. Rev. Lett, vol. 6, 1990.

[25] D. Anderson, Computational Fluid Mechanics and Heat Transfer. New York: McGraw-Hill Book Company, 1984.

[26] J. D. Anderson, Foundamentals of Aerodynamics. New York: McGraw-Hill Book Company, 1991.

[27] W. F. Ames, Numerical Methods for Partial Differential Equations. Israel: Dept. of Applied Mathematics, The Weizmann Institute of Science, 1984.

[28] W. Zhao, Z. Liu, and C. Liu, "Direct numerical simulation of flow transition in a supersonic airfoil boundary layer," in Proceedings of the First AFOSR International Conference on DNS/LES, Ruston, Louisiana, July 1997.

[29] E. Laurien and L. Kleiser, "Numerical simulation of boundary-layer transition and transition control," J. Fluid Mech., vol. 199, 1989.

[30] C. Liu and Z. Liu, "Multigrid mapping and box relaxation for simulation of the whole process of flow transition in 3-d boundary layers." J. Comp. Phys., vol. 119, 1995. 
[31] H. Shan, L. Jiang, W. Zhao, and C. Liu, "Secondary instability of wall-bounded shear flows," in AIAA paper, Reno, Nevada, Jan 1999.

[32] Z. Liu, G. Wang, and C. Liu, "Direct numerical simulation of flow transition in compressible swept-wing boundary layer," in Proceedings of the First AFOSR International Conference on DNS/LES, Ruston, Louisiana, July 1997.

[33] H. Schlichting, Boundary Layer Theory. New York: McGraw-Hill Book Company, 1979.

[34] X. Zhong and M. Tatineni, "High-order non-uniform grid schemes for numerical simulation of hypersonic boundary-layer stability and transition," Fluid Dynamics, vol. 190, 2003. 


\section{BIOGRAPHICAL STATEMENT}

Shutian Deng was born in Guangxi, P. R. China, in 1975. He received his B.S. and M.S. degree from Huazhong University of Science and Technology, China, in 1996 and 2000, respectively; his M.S. and Ph.D. degrees from The University of Texas at Arlington in 2003 and 2005, respectively, in Mathematics. His current research interest is in the area of various applications of the high order Discontinuous Galerkin FEM. He is a student member of AIAA. 